\title{
The Homology of Double Loop Spaces of Complex Stiefel Manifolds
}

By

\author{
Atsushi YAMAGUCHI*
}

\begin{abstract}
The Hopf algebra structure of $H_{*}\left(\Omega^{2} S U(n+1) / S U(m+1): \mathbb{F}_{p}\right)$ and the action of the Steenrod algebra on it are determined.
\end{abstract}

\section{Introduction}

Let $A$ be a primitively generated commutative Hopf algebra over a perfect field $K$ of characteristic $p$. Then, by Borel's theorem ([1]), $A$ is isomorphic to a tensor product of monogenic Hopf algebras. Using Künneth Formula, calculation of the cohomology of $A$ reduces to calculation of the cohomology of monogenic Hopf algebras. Let us denote by $V_{n, m}$ the complex Stiefel manifold $S U(n+1) / S U(m+1)$ and let $C_{n, m}$ be the mod $p$ ordinary homology of $\Omega V_{n, m^{*}}$ Since $\Omega V_{n, m}$ is a Hopf space, $C_{n, m}$ has a structure of Hopf algebra. In this case, $C_{n, m}$ is commutative and cocommutative, and we define a certain filtration of $C_{n, m}$ analogous to that of $S(n)_{*}$ in [5] so that the dual of the associate graded Hopf algebra is primitively generated. Then we can calculate $\operatorname{Cotor}_{*, *}^{R^{0} c_{n, m}}\left(\mathbb{F}_{p}, \mathbb{F}_{p}\right)$ since it is easy to calculate the cohomology of monogenic Hopf algebras. Showing that the spectral sequence associated with the filtration of $C_{n, m}$ collapses, we determine the $E^{2}$-term of the Eilenberg-Moore spectral sequence associated with the path fibration over $\Omega V_{n, m^{\circ}}$ On the other hand, a splitting of $C_{n, m}$ enable us to describe explicit cocycles of the cobar complex of $C_{n, m}$ which represent generators of $\operatorname{Cotor}_{*, *}^{C_{n, m} m}\left(\mathbb{F}_{p}, \mathbb{F}_{p}\right)$, then we can determine the differentials of the "algebraic" Bockstein spectral sequence and

Communicated by N. Shimada, February 3, 1986. Revised March 13, 1986.

* Department of Mathematics, University of Osaka Prefecture, Osaka 591, Japan. 
the Hopf algebra structure of $\operatorname{Cotor}_{*, *}^{C_{n, m}}\left(\mathbb{F}_{p}, \mathbb{F}_{p}\right)$.

The Hopf algebra structure of $\operatorname{Cotor}_{*, *}^{c} c_{n, m}\left(\mathbb{F}_{p}, \mathbb{F}_{p}\right)$ implies that the Eilenberg-Moore spectral sequence collapses, and $H_{*}\left(\Omega^{2} V_{n, m} ; \mathbb{F}_{p}\right)$ is given as follows if $p$ is an odd prime (See (4.14), (4.16) for details);

$$
\begin{gathered}
H_{*}\left(\Omega^{2} V_{n, m} ; \mathbb{F}_{p}\right)=E\left(h_{i, j} \mid m+1 \leqq i \leqq n, p \nmid i \text { or } i \leqq m p, j \geqq 0\right) \\
\otimes \mathbb{F}_{p}\left[g_{i, j} \mid m+1 \leqq i \leqq n, p \nmid i \text { or } i \leqq m p, j \geqq 0\right],
\end{gathered}
$$

where $\operatorname{deg} h_{i, j}=2 i p^{j}-1 \quad$ and $\operatorname{deg} g_{i, j}=2 i p^{j+e(n, i)+1}-2 \quad(e(n, i)=\max$ $\left.\left\{t \mid i p^{t} \leqq n\right\}\right)$ and $h_{i, j}$ and $g_{i, j}$ are primitive. Moreover, $h_{i, j}$ and $g_{i, j}$ are transgressive.

Section 1 is devoted to calculate the cohomology of monogenic Hopf algebras by constructing the minimal resolutions, and we examine induced mappings between the cohomology of monogenic Hopf algebras. In Section 2, we apply the results of Section 1 to calculation of the $E^{2}$-term of the Eilenberg-Moore spectral sequence associated with the path fibration over $\Omega V_{n, m}$. We examine the $E^{2}$-term in detail in Section 3, applying a splitting of $H_{*}\left(\Omega S U ; \mathbb{Z}_{(p)}\right)$. We find explicit cycles in the cobar complex which represent generators of the $E^{2}$-term and determine the differentials of the (algebraic) Bockstein spectral sequence of the $E^{2}$-term. We prove in Section 4 that the spectral sequence collapses and describe the Hopf algebra structure of $H_{*}\left(\Omega^{2} V_{n, m} ; \mathbb{F}_{p}\right)$ and morphisms induced by the canonical inclusion $V_{n, m} \subset V_{n+1, m}$ and projection $V_{n, m} \rightarrow V_{n, m+}{ }^{1}$. We also determine the homology suspensions $\sigma_{*}: H_{*}\left(\Omega^{2} V_{n, m} ; \mathbb{F}_{p}\right) \rightarrow H_{*+1}\left(\Omega V_{n, m} ; \mathbb{F}_{p}\right), \sigma_{*}: H_{*}$ $\left(\Omega^{3} V_{n, m} ; \mathbb{F}_{p}\right) \rightarrow H_{*+1}\left(\Omega^{2} V_{n, m} ; \mathbb{F}_{p}\right)$. In Section 5 , the Bockstein spectral sequence of $H_{*}\left(\Omega^{2} V_{n, m} ; \mathbb{F}_{p}\right)$ is examined. Finally, we determine the action of the Steenrod algebra on $H_{*}\left(\Omega^{2} V_{n, m} ; F_{p}\right)$ in Section 6.

Throughout this paper, we denote by $H_{*}(-)$ the mod $p$ ordinary homology unless otherwise stated and the modifications of statements required in the case $p=2$ are indicated inside square brackets.

I am indebted to Daniel Waggoner who showed me his results on $H_{*}\left(\Omega^{2} S U(n) ; F_{2}\right)$. He uses the Serre spectral sequence associated with a fibering $\Omega^{2} S U(n) \rightarrow \Omega^{2} S U(n+1) \rightarrow \Omega^{2} S^{2 n+1}$ to calculate $H_{*}\left(\Omega^{2} S U(n) ; \mathbb{F}_{2}\right)$. And I would like to thank Frederick Cohen, Douglas Ravenel and Stephen Wilson for helpful conversations. I would also like to express my gratitude to Akira Kono for his suggestions and for reading my 
messy manuscript.

I am deeply grateful to the referee for reading the manuscript very carefully and pointing out many errors.

\section{$\S 1$. Cohomology of Monogenic Hopl Algebras}

Let $K$ be a field of characteristic $p \neq 0$. We denote by $A(n, r)$ $(n \geqq 1)$ the monogenic graded Hopf algebra over $K$ generated by $x$ whose height is $p^{n}$ and $\operatorname{deg} x=2 r \quad[\operatorname{deg} x=r]$; that is, $A(n, r)=$ $K[x] /\left(x^{p^{n}}\right)$.

Let $E$ be a bigraded exterior algebra over $K$ generated by a single element $h$ having bidegree $(1,2 r)[(1, r)]$. And let $\Gamma$ be a bigraded divided polynomial algebra over $K$ spanned by $\left\{1=\gamma_{0}, \gamma_{1}, \gamma_{2}, \ldots\right.$, $\left.\gamma_{i}, \ldots\right\}$ with relations $\gamma_{i} \gamma_{j}=\left(\begin{array}{c}i+j \\ i\end{array}\right) \gamma_{i+j}$. Each $\gamma_{i}$ has bidegree $\left(2 i, 2 i r p^{n}\right)$ $\left[\left(2 i, 2^{n} i r\right)\right]$. We also assign $(0,2 r)[(0, r)]$ to $x$ in $A(n, r)$. Consider a bigraded $A(n, r)$-algebra $X(n, r)=A(n, r) \otimes E \otimes \Gamma$. We define a differential $d: X(n, r) \rightarrow X(n, r)$, a coproduct $\varphi: X(n, r) \rightarrow X(n, r) \otimes X(n$, $r$ ) and an augmentation $\varepsilon: X(n, r) \rightarrow K$ as follows;

$$
\begin{aligned}
& d\left(h \gamma_{i}\right)=x \gamma_{i}, d\left(\gamma_{i}\right)=x^{p^{n}-1} h \gamma_{i-1} \\
& \text { and } d \text { is an } A(n, r) \text {-linear map. } \\
& \varphi\left(h \gamma_{i}\right)=\sum_{j+k=i}\left(h \gamma_{j} \otimes \gamma_{k}+\gamma_{j} \otimes h \gamma_{k}\right), \\
& \varphi\left(\gamma_{i}\right)=\sum_{j+k=i} \gamma_{j} \otimes \gamma_{k}+\sum_{\substack{s+t=n^{n}-2 \\
j+k=i-1}}(-1)^{t} x^{s} h \gamma_{j} \otimes x^{t} h \gamma_{k}
\end{aligned}
$$

and $\varphi$ is an $A(n, r)$-linear map where the $A(n, r)$-module structure of $X(n, r) \otimes X(n, r)$ is the usual one, using coproduct $A(n, r) \rightarrow A(n, r) \otimes A(n, r)$

$$
\varepsilon(1)=1, \varepsilon\left(h \gamma_{i}\right)=\varepsilon\left(\gamma_{i}\right)=0 \text { and } \varepsilon \text { is } A(n, r) \text {-linear. }
$$

It is easy to verify that $X(n, r)$ is a differential Hopf algebra over $A(n, r)$. We also define a contracting homotopy

$$
\begin{aligned}
& s: X(n, r) \rightarrow X(n, r) \text { by } s\left(x^{i} \gamma_{j}\right)= \begin{cases}0, & \mathrm{i}=0 \\
x^{i-1} h \gamma_{j}, & 1 \leqq i \leqq p^{n}-1\end{cases} \\
& s\left(x^{i} h \gamma_{j}\right)=\left\{\begin{array}{ll}
0, & 0 \leqq i<p^{n}-1 \\
\gamma_{j+1}, & i=p^{n}-1
\end{array} \text { and let } \eta\right. \text { be the unit. }
\end{aligned}
$$

Then we have $d s+\eta \varepsilon=1, d s+s d=1$. Therefore $K \stackrel{\varepsilon}{\longleftarrow} X(n, r)$ is an $A$ $(n, r)$-free resolution of $K$. It is obvious that the complex $\operatorname{Hom}_{A(n, r)}$ 
$(X(n, r), K)$ has a trivial differential and it is isomorphic to $E\left(h^{*}\right)$ $\otimes K\left[g^{*}\right]\left[E\left(h^{*}\right) \otimes K\left[g^{*}\right]\right.$ for $n>1, K\left[h^{*}\right]$ for $\left.n=1\right]$ as an algebra, where $h^{*}$ and $g^{*}$ are the duals of $h$ and $\gamma_{1}$ respectively. Thus we obtain the following basic theorem.

Theorem 1.4. $\operatorname{Ext}_{A(n, r)}^{* * *}(K, K) \cong E\left(h^{*}\right) \otimes K\left[g^{*}\right]$ where

bideg $h^{*}=(1,2 r)$, bideg $g^{*}=\left(2,2 r p^{n}\right)$

$\left[\operatorname{Ext}_{A(n, r)}^{* * *}(K, K) \cong E\left(h^{*}\right) \otimes K\left[g^{*}\right]\right.$ for $n>1, \operatorname{Ext}_{A(1, r)}^{* * *}(K, K) \cong K\left[h^{*}\right]$, where

bideg $h^{*}=(1, r)$, bideg $\left.g^{*}=\left(2,2^{n} r\right)\right]$.

Let $A(\infty, r)$ be the monogenic Hopf algebra $K[x]$ (deg $x=2 r$ $[\operatorname{deg} x=r])$, then $X(\infty, r)=A(\infty, r) \otimes E$ with a differential $d(h)=x$ gives an $A(\infty, r)$-free minimal resolution of $K$. Let $h^{*}$ be the dual of $h$, then we have

Proposition 1.5. $\operatorname{Ext}_{A(\infty, r)}^{* * *}(K, K)=E\left(h^{*}\right)$ where bideg $h^{*}=(1,2 r)$ [bideg $\left.h^{*}=(1, r)\right]$.

There is another monogenic Hopf albegra $E(y)$ (deg $y=2 r-1$ ) over a field of odd characteristic. This case, $E(y) \otimes \Gamma$ (bideg $\gamma_{i}=$ $(i,(2 r-1) i))$ with a differential $d\left(\gamma_{i}\right)=y \gamma_{i-1}$ gives an $E(\gamma)$-free minimal resolution of $K$. Let $g^{*}$ be the dual of $\gamma_{1}$, then we get the following.

Proposition 1.6. $\operatorname{Ext}_{E(y)}^{* *}(K, K)=K\left[g^{*}\right]$ where bideg $g^{*}=(1,2 r-1)$.

Remark 1.7. Let $A(n, r)^{*}(1 \leqq n \leqq \infty)$ be the dual Hopf algebra of $A(n, r) . A(n, r)^{*}$ is spanned by $\left\{1=x_{0}, x_{1}, \ldots, x_{p^{n}-1}\right\}$ over $k^{-}$ with relations $x_{i} x_{j}=\left\{\begin{array}{lr}\left(\begin{array}{c}i+j \\ i\end{array}\right) x_{i+j}, i+j<p^{n} \\ 0, & i+j \geqq p^{n}\end{array}\right.$ and with a coproduct $\Delta x_{i}=$ $\sum_{j+k=i} x_{j} \otimes x_{k}$, where $x_{i}$ is the dual of $x^{i} \in A(n, r)$. The representations of $h^{*}$ and $g^{*}$ in the cobar complex $\Omega^{*}\left(A(n, r)^{*}\right)$ are given by $\left[x_{1}\right]$ and $\sum_{i=1}^{p^{n}-1}\left[x_{i} \mid x_{p^{n}-i}\right]$ respectively. It is straightforward to verify that both $h^{*}$ and $g^{*}$ are primitive in $\operatorname{Ext}_{A(n, r)}^{* * *}(K, K)$.

Let $\iota: A(n, r) \rightarrow A(m, r) \quad(1 \leqq m<n \leqq \infty)$ be a map of graded Hopf 
algebras defined by $\iota(x)=x$ and let $\pi: A\left(n, r p^{k}\right) \rightarrow A(k+n, r)(1 \leqq n \leqq \infty$, $1 \leqq k<\infty)$ be a map of graded Hopf algebras defined by $\pi(x)=x^{p^{k}}$. Then $\iota$ and $\pi$ induce maps of algebras $\iota^{\#}: \operatorname{Ext}_{A(m, r)}^{*, *}(K, K) \rightarrow \operatorname{Ext}_{A(n, r)}^{*, *}(K$, $K)$ and $\pi^{\sharp}: \operatorname{Ext}_{A(k+n, r)}^{*, *}(K, K) \rightarrow \operatorname{Ext}_{A\left(n, r p^{k}\right)}^{*}(K, K)$ respectively.

Lemma 1.8. $\iota^{\#}$ is given by $\iota^{\sharp}\left(h^{*}\right)=h^{*}$ and $\iota^{\sharp}\left(g^{*}\right)=0$ and $\pi^{\sharp}$ is given by $\pi^{\sharp}\left(h^{*}\right)=0$ and $\pi^{\sharp}\left(g^{*}\right)=g^{*}\left[\pi^{\sharp}\left(g^{*}\right)=\left(h^{*}\right)^{2}\right.$ if $\left.n=1\right]$ for $n<\infty$, and $\pi^{\sharp}$ $\left(h^{*}\right)=0$ for $n=\infty$.

Proof. ¿ induces a map of complexes over $K \iota_{\sharp}: X(n, r) \rightarrow X(m, r)$ such that $\iota_{\sharp}(h)=h, \iota_{\sharp}\left(\gamma_{i}\right)=0$ and $\iota_{\sharp}$ is a map of $A(n, r)$-Hopf algebras, where $X(m, r)$ is an $A(n, r)$-Hopf algebra via c. Taking the dual of $\iota_{\#,}$, it is straightforward to see that $\iota^{\sharp}\left(h^{*}\right)=h^{*}$ and $\iota^{\sharp}\left(g^{*}\right)=0$. If $n<\infty$, $\pi^{\sharp}$ induces a map of complexes over $K \pi_{\sharp}: X\left(n, r p^{k}\right) \rightarrow X(k+n, r)$ such that $\pi_{\#}(h)=x^{p^{k}-1} h, \pi_{\#}\left(\gamma_{i}\right)=\gamma_{i}$ and $\pi_{\#}$ is a map of $A\left(n, r p^{k}\right)-$ Hopf algebras. Taking the dual, we get the result. The case $n=\infty$ is easy。

\section{§2. Calculation of $\operatorname{Cotor}^{H}{ }^{\left(\Delta V_{n, m}\right)}\left(\mathbb{F}_{p}, \mathbb{F}_{p}\right)$}

Let $V_{n, m}(n>m)$ be the complex Stiefel manifold $S U(n+1) / S U(m+$ 1). Put $C_{n, m}=H_{*}\left(\Omega V_{n, m}\right)$, then it is known that $C_{n, m}$ is isomorphic to $\mathbb{F}_{p}\left[y_{m+1}, y_{m+2}, \ldots, y_{n}\right]\left(\operatorname{deg} y_{i}=2 i\right)$ as an algebra and the coproduct $\varphi$ is given by

$$
\varphi\left(y_{i}\right)= \begin{cases}1 \otimes y_{i}+y_{i} \otimes 1, & m+1 \leqq i \leqq 2 m+1 \\ 1 \otimes y_{i}+y_{i} \otimes 1+\sum_{\substack{k+l=i \\ k, l \geqq m+1}} y_{k} \otimes y_{l}, & 2 m+2 \leqq i \leqq n\end{cases}
$$

Define an increasing filtration $\left\{F_{i}\right\}$ of $C_{n, m}$ compatible with both product and coproduct by $y_{i}^{p^{j}} \in F_{i}-F_{i-1}$. Consider the associated graded Hopf algebra $E^{0} C_{n, m}$ and let $y_{i, j} \in E_{i}^{0} G_{n, m}$ be the class of $y_{i}^{p^{j}} \in F_{i}$. Then $E^{0} C_{n, m}$ is isomorphic to $\mathbb{F}_{p}\left[y_{i, j} \mid m+1 \leqq i \leqq n, j \geqq 0\right] /\left(y_{i, j}^{p}\right)$ as an algebra and the coproduct is given by

$$
\varphi y_{i, j}= \begin{cases}1 \otimes y_{i, j}+y_{i, j} \otimes 1, & m+1 \leqq i \leqq 2 m+1 \\ 1 \otimes y_{i, j}+y_{i, j} \otimes 1+\sum_{\substack{k+l=i \\ k, l \geqq m+1}} y_{k, j} \otimes y_{l, j}, & 2 m+2 \leqq i \leqq n\end{cases}
$$

Note that the $p$-th power map of $E^{0} C_{n, m}$ is trivial. Using the exact sequence of Milnor-Moore ([4]), it follows that the canonical map $P E^{0} C_{n, m} \rightarrow Q E^{0} C_{n, m}$ is a monomorphism. Therefore the dual Hopf 
algebra $E^{0} C_{n, m}^{*}$ of $E^{0} C_{n, m}$ is primitively generated. In fact, the canonical map $P E^{0} C_{n, m}^{*} \rightarrow Q E^{0} C_{n, m}^{*}$ is an epimorphism, which is the dual of $P E^{0} C_{n, m} \rightarrow Q E^{0} C_{n, m}$. Take a basis of $E^{0} C_{n, m}$ which consists of monomials in $y_{i, j}$ 's and consider the dual basis. Let us denote by $y_{i, j}^{*}$ the dual of $y_{i, j}$. Then, $\left\{y_{i, j}^{*} \mid m+1 \leqq i \leqq n, j \geqq 0\right\}$ is a basis of $P E^{0} C_{n, m}^{*}$. Easy calculation shows an equality

$$
\left(y_{i, j}^{*}\right)^{p}=\left\{\begin{array}{ll}
y_{i p, j}^{*}, & i p \leqq n \\
0, & i p>n
\end{array} .\right.
$$

This proves the following lemma.

Lemma 2.1. $\left\{y_{i, j}^{*} \mid m+1 \leqq i \leqq n, p \nmid i\right.$ or $\left.i \leqq m p, j \geqq 0\right\}$ generates $E^{0} C_{n, m}^{*}$ as an algebra with relations $\left(y_{i, j}^{*}\right)^{p^{e(n, i)+1}}=0$ where $e(n, i)=\max \{t \in \mathbb{Z} \mid$ $\left.i p^{t} \leqq n\right\}$. Therefore $E^{0} C_{n, m}^{*}$ is isomorphic to

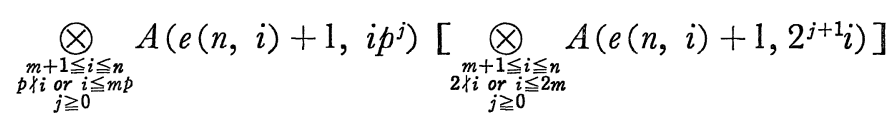

as a Hopf algebra.

Using Künneth formula and (1. 4-5), we have the following.

Lemma 2.2. $\operatorname{Cotor}_{*, *}^{E^{0} c_{n, m}}\left(F_{p}, F_{p}\right) \cong \operatorname{Ext}_{E^{0} C_{n, m}^{* * *}}^{-* *}\left(F_{p}, F_{p}\right) \cong E\left(h_{i, j} \mid m+1 \leqq\right.$ $i \leqq n, \quad p \nmid i$ or $i \leqq m p, j \geqq 0) \otimes \mathbb{F}_{p}\left[g_{i, j} \mid m+1 \leqq i \leqq n, p \nmid i\right.$ or $\left.i \leqq m p, j \geqq 0\right]$ [Cotor ${ }^{E^{0} C_{n, m}}\left(\mathbb{F}_{2}, F_{2}\right) \cong \mathrm{Ext}_{E^{0} C_{n, m}^{*} * *}^{-*}\left(\mathbb{F}_{2}, \mathbb{F}_{2}\right) \cong E\left(h_{i, j} \mid m+1 \leqq i \leqq\left[\frac{n}{2}\right], 2 \nmid i\right.$ or $i \leqq$ $2 m, j \geqq 0) \otimes F_{2}\left[h_{i, j} \mid \max \left\{m,\left[\frac{n}{2}\right]\right\}<i \leqq n, 2 \nmid i\right.$ or $\left.i \leqq 2 m, j \geqq 0\right] \otimes \mathbb{F}_{2}\left[g_{i, j} \mid m\right.$ $+1 \leqq i \leqq\left[\frac{n}{2}\right], 2 \nmid i$ or $\left.\left.i \leqq 2 m, j \geqq 0\right]\right]$ for $n<\infty$, and $\operatorname{Cotor}_{*, *}^{E^{0} C_{\infty, m}}\left(\mathbb{F}_{p}, \mathbb{F}_{p}\right)$ $\cong \operatorname{Ext}_{E^{0} C_{\infty, m}^{* * *}}^{-* * *}\left(\mathbb{F}_{p}, \mathbb{F}_{p}\right) \cong E\left(h_{i, j} \mid i \geqq m+1, p \nmid i\right.$ or $\left.i \leqq m p, j \geqq 0\right)$ for $n=\infty$, where bideg $h_{i, j}=\left(-1,2 i p^{j}\right)$, bideg $g_{i, j}=\left(-2,2 i p^{j+e(n, i)+1}\right)$. Here we adapted the grading for the Eilenberg-Moore spectral sequence.

Let $\iota_{n, m}: V_{n, m} \rightarrow V_{n+1, m}$ be the canonical inclusion and let $\pi_{n, m}: V_{n, m}$ $\rightarrow V_{n, m+1}$ be the canonical projection. By an abuse of notations, we also denote $\Omega^{k} \iota_{n, m}, \Omega^{k} \pi_{n, m}(k=1,2)$ by $c_{n, m}, \pi_{n, m}$ respectively. The induced maps $\iota_{n, m^{*}}: C_{n, m} \rightarrow C_{n+1, m}$ and $\pi_{n, m^{*}}: C_{n, m} \rightarrow C_{n, m+1}$ are given by

$$
\begin{aligned}
& \iota_{n, m^{*}}\left(y_{i}\right)=y_{i}(m+1 \leqq i \leqq n) \\
& \pi_{n, m^{*}}\left(y_{i}\right)=\left\{\begin{array}{ll}
0, & i=m+1 \\
y_{i}, & m+2 \leqq i \leqq n
\end{array} .\right.
\end{aligned}
$$

Since $\ell_{n, m}$ and $\pi_{n, m}$ preserve the filtrations on $C_{n, m}$ 's, they induce 
$\iota_{n, m^{*}}: E^{0} C_{n, m} \rightarrow E^{0} C_{n+1, m}$ and $\pi_{n, m^{*}}: E^{0} C_{n, m} \rightarrow E^{0} C_{n, m+1}$ which are obviously given by

$$
\begin{aligned}
& \iota_{n, m^{*}}\left(y_{i, j}\right)=y_{i, j} \quad(m+1 \leqq i \leqq n, j \geqq 0) \\
& \pi_{n, m^{*}}\left(y_{i, j}\right)= \begin{cases}0, & i=m+1, j \geqq 0 \\
y_{i, j}, & m+2 \leqq i \leqq n, j \geqq 0 .\end{cases}
\end{aligned}
$$

Taking the dual of $\iota_{n, m^{*}}$ and $\pi_{n, m^{*}}$, we have maps $\iota_{n, m}^{*}: E^{0} C_{n+1, m}^{*}$ $\rightarrow E^{0} C_{n, m}^{*}$ and $\pi_{n, m}^{*}: E^{0} C_{n, m+1}^{*} \rightarrow E^{0} C_{n, m}^{*}$ which map $y_{i, j}^{*}$ 's as follows.

$$
\begin{aligned}
& \iota_{n, m}^{*}\left(y_{i, j}^{*}\right)=\left\{\begin{array}{lll}
y_{i, j}^{*}, & m+1 \leqq i \leqq n & j \geqq 0 \\
0, & i=n+1 & j \geqq 0
\end{array},\right. \\
& \pi_{n, m}^{*}\left(y_{i, j}^{*}\right)=y_{i, j}^{*}(m+2 \leqq i \leqq n, j \geqq 0) .
\end{aligned}
$$

Consider the case $p \mid n+1$ and $n \geqq m p$, and take an integer $s(n, m)$ $=\max \left\{s \mid(m+1) p^{s} \leqq n+1\right.$ and $\left.p^{s} \mid n+1\right\}$. Then $s(n, m) \geqq 1$ and $\iota_{n, m}^{*}$ $\left(y_{k(n, m), j}^{*}\right)^{p^{s(n, m)}}=0$, where $k(n, m)=\frac{n+1}{p^{s(n, m)}}$. On the other hand, we have $\left(y_{k(n, m), j}^{*}\right)^{p^{s(n, m)}}=y_{n+1, j}^{*} \neq 0$. Next, consider the case $n \geqq(m+1) p$, then $\pi_{n, m}^{*}\left(y_{(m+1) p, j}^{*}\right)=\left(y_{m+1, j}^{*}\right)^{p}$.

By the above observations, we obtain the following lemma, applying (1.8) and (2.1).

Lemma 2.6. $\iota_{n, m}$ and $\pi_{n, m}$ induce maps $\iota_{n, m}^{\sharp}:$ Cotor ${ }_{*, *}^{E^{0} C_{n, m}}\left(\mathbb{F}_{p}, \mathbb{F}_{p}\right) \rightarrow$ $\operatorname{Cotor}_{*, *}^{E^{0} C_{n, m}}\left(\mathbb{F}_{p}, \mathbb{F}_{p}\right)$ and $\pi_{n, m}: \operatorname{Cotor}_{*, *}^{E^{0} C_{n, m}}\left(\mathbb{F}_{p}, \mathbb{F}_{p}\right) \rightarrow \operatorname{Cotor}_{*, *}^{E^{0} C_{n, m+1}}\left(\mathbb{F}_{p}, \mathbb{F}_{p}\right)$ which are described as follows.

$\ell_{n, m} \#\left(h_{i, j}\right)=h_{i, j}$ in any cases.

$\iota_{n, m^{\sharp}}\left(g_{i, j}\right)=\left\{\begin{array}{l}0, p \mid n+1 \text { and } n \geqq m p \text { and } i=k(n, m) \\ g_{i, j}, \text { otherwise }\end{array}\right.$

$\left[\ell_{n, m}\left(g_{i, j}\right)=\left\{\begin{array}{l}0,4 \mid n+1 \text { and } n \geqq 4 m \text { and } i=k(n, m) \\ g_{i, j}, \text { otherwise }\end{array}\right]\right.$

$\pi_{n, m}^{\#}\left(h_{i, j}\right)= \begin{cases}0, & i=m+1 \\ h_{i, j}, & i \neq m+1\end{cases}$

$\pi_{n, m^{\sharp}}\left(g_{i, j}\right)=\left\{\begin{array}{lll}g_{i, j}, & i \neq m+1 \\ g_{(m+1) p, j}, & i=m+1, & (m+1) p \leqq n \\ 0, & i=m+1, & (m+1) p>n\end{array}\right.$

$\left[\pi_{n, m^{\sharp}}\left(g_{i, j}\right)=\left\{\begin{array}{ll}g_{i, j}, & i \neq m+1 \\ g_{2(m+1), j}, & i=m+1, n \geqq 4(m+1) \\ h_{2(m+1), j}^{2}, & i=m+1,2(m+1) \leqq n<4(m+1)\end{array}\right]\right.$ 
Remark 2.7. It is easy to see that the map $\operatorname{Cotor}_{*, *}^{E^{0} C_{n, m}}\left(\boldsymbol{F}_{p}, \boldsymbol{F}_{p}\right)$ $\rightarrow \operatorname{Cotor}_{*, *}^{E^{0} C_{\infty, m}}\left(\boldsymbol{F}_{p}, \boldsymbol{F}_{p}\right)$ induced by the canonical inclusion $\Omega V_{n, m} \rightarrow \Omega V_{\infty, m}$ maps $h_{i, j}$ to $h_{i, j}$ and $g_{i, j}$ to zero.

The filtration of $C_{n, m}$ defines a filtration of the cobar complex $\Omega_{*}\left(C_{n, m}\right)$; that is

$$
F_{s} \Omega_{k}\left(C_{n, m}\right)=\sum_{s_{1}+\cdots+s_{-k}=s-k} F_{s_{1}} C_{n, m} \otimes \ldots \otimes F_{s_{-k}} C_{n, m}(k \leqq 0)
$$

Then we have $E_{s, t, u}^{0}=\left(F_{s} \Omega_{s+t}\left(C_{n, m}\right) / F_{s-1} \Omega_{s+t}\left(C_{n, m}\right)\right)_{u+t} \cong$

$$
\Omega_{s+t}\left(E^{0} C_{n, m}\right)_{-t, u+t}
$$

Consider the spectral sequence associated with this filtration. Note that this spectral sequence is trigraded and its $E^{2}$-term is given by

$$
\begin{aligned}
E_{s, t, u}^{2}=\operatorname{Cotor}_{s+t,-t, u+t}^{E^{0} C_{n, m}}\left(\boldsymbol{F}_{p}, \boldsymbol{F}_{p}\right) \text { and } h_{i, j} & \in \sum_{s+t=-1} E_{s, t, *}^{2}, \\
g_{i, j} & \in \sum_{s+t=-2} E_{s, t, *}^{2}
\end{aligned}
$$

In the case $n=\infty . \quad E_{*, *, *}^{2}$ is an exterior algebra generated by $h_{i, j}$ which belongs to $\sum_{s+t=-1} E_{s, t, *}^{2}$. By the remark (1.7), these $h_{i, j}$ 's are primitive and there are not any primitive elements in $\sum_{s+t=-2} E_{s, t, *}^{2}$. This implies that the spectral sequence $\operatorname{Cotor}_{*, *}^{E^{0} C_{\infty, m}}\left(\mathbb{F}_{p}, \mathbb{F}_{p}\right) \Rightarrow \operatorname{Cotor}_{*, *}^{C_{\infty}, m}$ $\left(\boldsymbol{F}_{p}, \boldsymbol{F}_{p}\right)$ collapses. Let $h_{i, j} \in \operatorname{Cotor}_{-1,2 i p^{j}}^{C_{\infty, m}}\left(\boldsymbol{F}_{p}, \boldsymbol{F}_{p}\right)$ be the element which corresponds to $h_{i, j}$ in Cotor ${ }_{-i, i, 2 i p^{j}-i}^{E^{0} C_{\infty_{i} m}}\left(\mathbb{F}_{p}, \boldsymbol{F}_{p}\right)$.

Lemma 2.10. The extension is trivial: that is $\operatorname{Cotor}_{*, *}^{C_{\infty, m}}\left(\boldsymbol{F}_{p}, \boldsymbol{F}_{p}\right)$ $=E\left(h_{i, j} \mid i \geqq m+1, p \nmid i\right.$ or $\left.i \leqq m p, j \geqq 0\right)$

Proof. If $p$ is odd, it is obvious that $h_{i, j}^{2}=0$ in $\operatorname{Cotor}_{*, *}^{C_{\infty, m}}\left(\boldsymbol{F}_{p}, \boldsymbol{F}_{p}\right)$ because the total degree of $h_{i, j}$ is odd. Let us consider the case $p=2$. If $m=0$, Cotor ${ }_{*, *}^{C_{\infty}, 0}\left(\mathbb{F}_{2}, F_{2}\right)$ is the $E^{2}$-term of the Eilenberg-Moore spectral sequence conversing to $H_{*}\left(\Omega^{2} S U\right)$. By Bott periodicity, $\Omega^{2} S U$ is homotopy equivalent to $U$ whose homology is isomorphic to $E\left(h_{1}, h_{2}, \ldots\right)$ (deg $\left.h_{i}=2 i-1\right)$. Comparing the Poincare series of $H_{*}\left(\Omega^{2} S U\right)$ with that of Cotor $C_{*, *}^{C_{\infty, m}}\left(\boldsymbol{F}_{2}, \boldsymbol{F}_{2}\right)$, the Eilenberg-Moore spectral sequence collapses. Since squaring map of $E^{\infty}=E^{2}$-term is also trivial. Thus we have $h_{i, j}^{2}=0$ in $\operatorname{Cotor}_{*, *}^{c_{\infty, 0}}\left(\mathbb{F}_{2}, \mathbb{F}_{2}\right)$. Since $h_{i, j}(i \geqq$ $m+1,2 \nmid i)$ in $\operatorname{Cotor}_{*, *}^{C_{\infty, m}}\left(\boldsymbol{F}_{2}, \boldsymbol{F}_{2}\right)$ is in the image of the map induced 
by $S U \rightarrow S U / S U(m+1)$ from Lemma 2.6 , we have $h_{i, j}^{2}=0$ for $i \geqq m$ +1 and $2 \nmid i$. So we only have to prove that $h_{i, j}^{2}=0$ for $\mathrm{m}+1 \leqq i \leqq 2 m$. It is easy to see that the representative of $h_{i, j}$ in the cobar complex $\Omega_{*}\left(G_{\infty, m}\right)$ is $\left[y_{i}^{2^{j}}\right]$. And it is also easy to verify the formula

$$
\begin{aligned}
& d\left(\left[y_{2 i}^{2^{j}}\right]+\sum_{k=0}^{i-m-2}\left[y_{m+1+k}^{2^{j}} y_{2 i-m-1-k}^{2^{j}}\right]+\sum_{k=0}^{[i-i(m+1)]}\left[y_{m+1+k}^{2^{j+1}} y_{2 i-2 m-2-2 k}^{2^{j}}\right]\right)= \\
& {\left[y_{i}^{2^{j}} \mid y_{i}^{2^{j}}\right], \text { which implies } h_{i, j}^{2}=0 \text { for } m+1 \leqq i \leqq 2 m .}
\end{aligned}
$$

To prove that the spectral sequence $\operatorname{Cotor}_{*, *}^{E^{0} C_{n, m}}\left(\mathbb{F}_{p}^{*}, \mathbb{F}_{p}\right) \Rightarrow$ Cotor $_{*, *}^{C_{n, m}}$ $\left(F_{p}, F_{p}\right)$ collapses, we use the Frobenius map $F$ which is induced by the $p$-th power map of $C_{n, m}$. Since the $p$-th power map of $C_{n, m}$ preserves the filtration, the Frobenius map $F$ of $\Omega_{*}\left(C_{n, m}\right)$ also preserves the filtration of $\Omega_{*}\left(C_{n, m}\right)$ where $F$ sends $\left[x_{1}|\ldots| x_{s}\right]$ to $\left[x_{1}^{p}|\ldots| x_{s}^{p}\right]$. Clearly $F$ commutes with the differential of $\Omega_{*}\left(C_{n, m}\right)$. Therefore $F$ induces a map of the spectral sequence.

Lemma 2.11. The spectral sequence $\operatorname{Cotor}_{*, *}^{E^{0} C_{n, m}}\left(\mathbb{F}_{p}, \mathbb{F}_{p}\right) \Rightarrow$ Cotor $_{*, *}^{C_{n} m}$ $\left(F_{p}, F_{p}\right)$ collapses.

Proof. Since the inclusion map $V_{n, m} \rightarrow V_{\infty, m}$ induces an isomorphism $C_{n, m} \rightarrow C_{\infty, m}$ for degree $\leqq 2 n+1, \Omega_{*}\left(C_{n, m}\right)_{t} \rightarrow \Omega_{*}\left(C_{\infty, m}\right)_{t}$ is an isomorphism for $t \leqq 2 n+1$. This yields that the induced map $\operatorname{Cotor}_{*, t}^{c_{n, m}}\left(\mathbb{F}_{p}, \mathbb{F}_{p}\right) \rightarrow$ $\operatorname{Cotor}_{*, i}^{C_{\infty} m}\left(\mathbb{F}_{p}, F_{p}\right)$ is an isomorphism for $t \leqq 2 n+1$, and thus we see

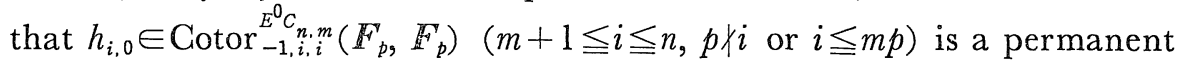
cycle by considering the map between the two spectral sequences. Noting that the Frobenius map $F$ of $\operatorname{Cotor}_{*, *}^{E^{0} C_{n, m}}\left(\mathbb{F}_{p}, \mathbb{F}_{p}^{\prime}\right)$ maps $h_{i, j}$ to $h_{i, j+1}$, we see that $h_{i, j}$ 's in $\operatorname{Cotor}_{*, *}^{E^{0} C_{n, m}}\left(\mathbb{F}_{p}, \mathbb{F}_{p}\right)$ are all permanent cycles. Note that the spectral sequence has a structure of a differential Hopf algebra and that $g_{i, j}$ 's are all primitive by (1.7). Moreover, there is no primitive element in $\sum_{s+t=-3} E_{s, t, *}^{2}$. Hence $g_{i, j}$ 's are also permanent cycles.

Lemma 2.12. The extension of the spectral sequence $\operatorname{Cotor}_{*, *}^{E^{0} C_{n, m}}\left(\mathbb{F}_{p}, \mathbb{F}_{p}\right)$ $\Rightarrow \operatorname{Cotor}_{*, *}^{C}\left(\mathbb{F}_{p}, \mathbb{F}_{p}\right)$ is trivial. Thus we have

$$
\operatorname{Cotor}_{*, *}^{c_{n, m}}\left(\mathbb{F}_{p}, \mathbb{F}_{p}\right)=E\left(h_{i, j} \mid m+1 \leqq i \leqq n, p \nmid i \text { or } i \leqq m p, j \geqq 0\right)
$$


$\otimes \boldsymbol{F}_{p}\left[g_{i, j} \mid m+1 \leqq i \leqq n, p \nmid i\right.$ or $\left.i \leqq m p, j \geqq 0\right]$

$\left[\operatorname{Cotor}_{*, *}^{c} c_{n, m}\left(\mathbb{F}_{2}, \mathbb{F}_{2}\right)=E\left(h_{i, j} \mid m+1 \leqq i \leqq\left[\frac{n}{2}\right], 2 \nmid i\right.\right.$ or $\left.i \leqq 2 m, j \geqq 0\right)$

$\otimes \boldsymbol{F}_{2}\left[h_{i, j} \mid \max \left\{\left[\frac{n}{2}\right], m\right\}<i \leqq n, 2 \nmid i\right.$ or $\left.i \leqq 2 m, j \geqq 0\right]$

$\otimes \mathbb{F}_{2}\left[g_{i, j} \mid m+1 \leqq i \leqq\left[\frac{n}{2}\right], 2 \nmid i\right.$ or $\left.\left.i \leqq 2 m, j \geqq 0\right]\right]$,

where $h_{i, j}$ and $g_{i, j}$ are the elements corresponding to the permanent cycles $h_{i, j}$ and $g_{i, j}$ in the $E^{2}$-term and bideg $h_{i, j}=\left(-1,2 i p^{j}\right)$, bideg $g_{i, j}=(-2$, $\left.2 i p^{j+e(n, i)+1}\right)$.

Proof. If $p$ is odd, triviality of the extension is obvious. Note that the Frobenius map of $\operatorname{Cotor}_{*, *}^{c_{n, m}}\left(\boldsymbol{F}_{p}, \boldsymbol{F}_{p}\right)$ maps $h_{i, j}$ to $h_{i, j+1}$ for any prime $p$. So it suffices to prove that $h_{i, 0}^{2}=0$ for $m+1 \leqq i \leqq\left[\frac{n}{2}\right]$ when $p=2$. Since bideg $h_{i, 0}^{2}=(-2,4 i)$ and $4 i \leqq 2 n$, recalling that Cotor ${ }_{*, t}^{c_{n, m}}$ $\left(F_{2}, F_{2}\right) \rightarrow \operatorname{Cotor}_{*, t}^{C_{\infty, m}}\left(F_{2}, F_{2}\right)$ is an isomorphism for $t \leqq 2 n$, we have $h_{i, 0}^{2}=0$ by $(2.10)$.

\section{§3. Splitting of $C_{n, m}$ and the Bockstein Spectral Sequence of $\operatorname{Cotor}_{*, *}^{C_{n, m}}\left(\mathbb{F}_{p}, F_{p}\right)$}

Husemoller proved in [3] that the Hopf algebra $C_{\infty, 0}$ decomposes as an infinite tension product of certain Hopf algebra on infinitely many generators. We give an explicit description of a splitting of $C_{n, m}$ in this section.

Let $\bar{G}_{n, m}$ be a Hopf algebra $\boldsymbol{Z}_{(p)}\left[y_{m+1}, y_{m+2}, \ldots, y_{n}\right] \quad\left(\operatorname{deg} y_{i}=2 i\right)$ whose coproduct is given by

$$
\varphi\left(y_{i}\right)= \begin{cases}1 \otimes y_{i}+y_{i} \otimes 1, & m+1 \leqq i \leqq 2 m+1 \\ 1 \otimes y_{i}+y_{i} \otimes 1+\sum_{\substack{k+l=i \\ k, l \geqq m+1}} y_{k} \otimes y_{l}, & 2 m+2 \leqq i \leqq n\end{cases}
$$

Hence $C_{n, m}=\bar{C}_{n, m} \otimes \mathbb{F}_{p}$. Let $f_{i} \in \bar{C}_{n, 0}\left(f_{i} \in C_{n, 0}\right)$ be the $i$-th Newton polynomial. That is, $f_{i}$ is defined inductively by $f_{1}=y_{1}$ and $f_{i}=$ $y_{1} f_{i-1}-y_{2} f_{i-2}+\ldots+(-1)^{i} y_{i-1} f_{1}+(-1)^{i+1} i y_{i}$. We also use the notation $f_{i}$ as a reduction of $f_{i} \in \bar{C}_{n, 0}\left(f_{i} \in C_{n, 0}\right)$ by a map $\bar{C}_{n, 0} \rightarrow \bar{C}_{n, m}\left(C_{n, 0} \rightarrow C_{n, m}\right)$.

Lemma 3.1. Let $a_{i, j}(p \nmid i, j \geqq 0)$ be the element of $p^{-1} \bar{C}_{\infty, 0}$ defined 
inductively by $a_{i, 0}=f_{i}$ and $a_{i, 0}^{p^{j}}+p a_{i, 1}^{p^{j-1}}+\ldots+p^{j} a_{i, j}=f_{i p^{j \cdot}}$ Then $a_{i, j} \in \bar{C}_{\infty, 0}$ $a_{i, j} \equiv(-1)^{i p^{j}+1} i y_{i p^{j}}$ modulo decomposables of $\bar{C}_{\infty, 0}$ and $\left\{a_{i, j} \mid p \nmid i, j \geqq 0\right\}$ generates $\bar{C}_{\infty, 00}$. Moreover the subalgebra $\bar{B}_{i}$ of $\bar{C}_{\infty, 0}$ generated by $\left\{a_{i, j} \mid j \geqq 0\right\}$ is a direct summand of $\bar{C}_{\infty, 0}$ and a sub Hopf algebra of $\bar{C}_{\infty, 0}$.

Proof. The proof of the first assertion is due to Ravenel ([5], Lemma 1.7). If $j=0, a_{i, 0}=f_{i} \in \bar{C}_{\infty, 0}$ Assume inductively that $a_{i, j}$ $\in \bar{C}_{\infty, 0}$ for $j=0,1, \ldots, r-1$. It suffices to prove that $\sum_{j=0}^{r-1} p^{j} a_{i, j}^{p^{r-j}} \equiv$ $f_{i p^{r}} \bmod p^{r}$. Each $a_{i, j}$ and $f_{i p^{j}}$ are polynomials of $y_{1}, y_{2}, \ldots, y_{i p^{j}}$ We consider $y_{k}\left(k=1,2, \ldots, i p^{r}\right)$ as the $k$-th elementary symmetric polynomial of indeterminates $t_{1}, t_{2}, \ldots, t_{N}\left(N=i p^{r}\right)$, then $a_{i, j}(j=0,1, \ldots$, $r-1)$ is a symmetric polynomial of $t_{1}, \ldots, t_{N}$ and we put $a_{i, j}=a_{i, j}$ $\left(t_{1}, \ldots, t_{N}\right) \in \bar{C}_{N, 0} \subset \mathbb{Z}_{(p)}\left[t_{1}, \ldots, t_{N}\right]$. By definition we have

$$
\sum_{j=0}^{r-1} p^{j} a_{i, j}\left(t_{1}, \ldots, t_{N}\right)^{p^{r-1-j}}=f_{i p^{r-1}}=\sum_{l=1}^{N} t_{l}^{i p^{r-1}} \ldots(*) \text {. }
$$

Noting that $a_{i, j}\left(t_{1}^{p}, \ldots, t_{N}^{p}\right) \equiv a_{i, j}\left(t_{1}, \ldots, t_{N}\right)^{p} \bmod p$, we have $p^{j} a_{i, j}$ $\left(t_{1}^{p}, \ldots, t_{N}^{p}\right)^{p^{r-1-j}} \equiv p^{j} a_{i, j}\left(t_{1}, \ldots, t_{N}\right)^{p^{r-j}} \bmod p^{r}$ (See (i) of (3.4) below). Replacing $t_{l}$ by $t_{l}^{p}$ in $(*)$, we have

$$
\sum_{j=0}^{r-1} p^{j} a_{i, j}\left(t_{1}, \ldots, t_{N}\right)^{p^{r-j}} \equiv \sum_{l=1}^{N} t_{l}^{i p^{r}}=f_{i p^{r}} \bmod p^{r} \text { in } \mathbb{Z}_{(p)}\left[t_{1}, \ldots, t_{N}\right] \text { 。 }
$$

Since $\bar{C}_{N, 0}$ is a direct summand of $\mathbb{Z}_{(p)}\left[t_{1}, \ldots, t_{N}\right]$, it follows that $\sum_{j=0}^{r-1} p^{j} a_{i, j}^{p^{r-j}} \equiv f_{i p^{r}} \bmod p^{r}$ in $\bar{C}_{N, 0}$. Therefore $a_{i, r} \in \bar{C}_{N, 0} \subset \bar{C}_{\infty, 0}$

The fact $f_{i} \equiv(-1)^{i+1} i y_{i}$ modulo decomposables implies $a_{i, j} \equiv$ $(-1)^{i p^{j}+1} i y_{i p^{j}}$. We prove that each $y_{k}$ is contained in the subalgebra generated by $\left\{a_{i, j} \mid p \nmid i, j \geqq 0\right\}$ by induction on $k$. Since $y_{1}=a_{1,0}$, the assertion is obvious if $k=1$. Assume that the assertion is true for $k=1,2, \ldots, l-1$. Putting $l=i p^{j}(p \nmid i, j \geqq 0)$, then $y_{l}=\frac{(-1)^{i p^{j}+1}}{i} a_{i, j}+$ $\sum_{s \geqq 1} \lambda_{s} Y_{s}$ where $\lambda_{s} \in \mathbb{Z}_{(p)}$ and $Y_{s}$ is a monomial of $y_{1}, \ldots, y_{l-1}$. By the assumption $y_{l}=\frac{(-1)^{i p^{j}+1}}{i} a_{i, j}+\sum_{t \geqq 1} \alpha_{t} A_{t}$ where $\alpha_{t} \in \mathbb{Z}_{(p)}$ and $A_{t}$ is a monomial of $a_{r, s}\left(r p^{s}<l\right)$. Thus the induction proceeds. Now we have $\bar{C}_{\infty, 0}=\mathbb{Z}_{(p)}\left[a_{i j} \mid p \nmid i, j \geqq 0\right]=\bigotimes_{p \nmid i} \bar{B}_{i}$ and the assertion that $\bar{B}_{i}$ is a direct summand of $\bar{C}_{\infty, 0}$ is obvious. Therefore, the equality $p^{-1} \bar{B}_{i} \cap \bar{C}_{\infty, 0}=\bar{B}_{i}$ holds.

Since $f_{i}$ is primitive in $\bar{C}_{\infty, 0}$, it is obvious that $\varphi a_{i, 0}=\varphi f_{i} \in \bar{B}_{i} \otimes \bar{B}_{i}$ 。 Assume inductively that $\varphi a_{i, j} \in \bar{B}_{i} \otimes \bar{B}_{i}$ for $j=0,1, \ldots, r-1$. Applying 
the coproduct to the defining formula of $a_{i, j}$, we have $p^{r} \varphi a_{i, r}=$ $1 \otimes f_{i p^{r}}+f_{i p^{r}} \otimes 1-\sum_{j=0}^{r-1} p^{j}\left(\varphi a_{i, j}\right)^{p^{r-j}}=1 \otimes\left(\sum_{j=0}^{r} p^{j} a_{i, j}^{p^{r-j}}\right)+\left(\sum_{j=0}^{r} p^{j} a_{i, j}^{p^{r-j}}\right) \otimes 1$

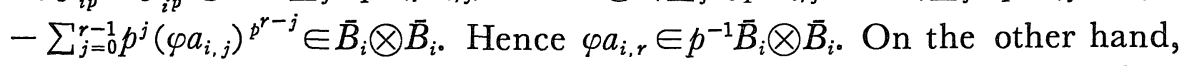
$\varphi a_{i, j} \in \bar{C}_{\infty, 0} \otimes \bar{C}_{\infty, 0}$ since $a_{i, j} \in \bar{C}_{\infty, 0 .}$ Thus $\varphi a_{i, j} \in\left(p^{-1} \bar{B}_{i} \otimes \bar{B}_{i}\right) \cap\left(\bar{C}_{\infty, 0} \otimes \bar{C}_{\infty, 0}\right)$ $=\bar{B}_{i} \otimes \bar{B}_{i}$ since $\bar{B}_{i}$ is a direct summand of $\bar{G}_{\infty, 0}$.

Let $\bar{B}_{i}(r, 0)$ be the sub Hopf algebra of $\bar{B}_{i}$ generated by $a_{i, 0}, a_{i, 1}$, $\ldots, a_{i, r}$ And let $\bar{B}_{i}(r, s)(0 \leqq s \leqq r+1)$ be the quotient Hopf algebra of $\bar{B}_{i}(r, 0)$ by the ideal generated by $a_{i, 0}, \ldots, a_{i, s-1}$. We put $B_{i}(r, s)$ $=\bar{B}_{i}(r, s) \otimes F_{p}$, and we also use $a_{i, j}$ to represent the reduction of $a_{i, j}$ $\in \bar{B}_{i}$ to $\bar{G}_{n, m}, C_{n, m}, \bar{B}_{i}(r, s)$ or $B_{i}(r, s)$.

Remark 3.2. Since $a_{i, j} \equiv(-1)^{i p^{j}+1} i y_{i p^{j}}$ modulo decomposables, the canonical map $\bar{B}_{i}(e(n, i), e(m, i)+1) \rightarrow{\stackrel{i \bar{C}^{j}}{n, m}}^{j}(1 \leqq i \leqq n, p \nmid i)$ which sends $a_{i, j}$ to $a_{i, j}$ is monomorphic, where we put $e(m, i)=-1$ if $i>m$. So we may regard $\bar{B}_{i}(e(n, i), e(m, i)+1)$ as a sub Hopf algebra of $\bar{C}_{n, m}$. Similarly we regard $B_{i}(e(n, i), e(m, i)+1)$ as a sub Hopf algebra of $C_{n, m}$.

The following is a direct consequence of (3.1).

Corollary 3.2. $\quad \bar{B}_{i}(r, s)\left(B_{i}(r, s)\right)$ is a polynomial algebra over $\mathbb{Z}_{(p)}$ (resp. $\mathbb{F}_{p}$ ) generated by $a_{i, s}, a_{i, s+1}, \ldots, a_{i, r}$, and we have the following splittings: $\bar{C}_{n, m}=\bigotimes_{\substack{p \nmid i \\ 1 \leqq i \leqq n}} \bar{B}_{i}(e(n, i), e(m, i)+1), C_{n, m}=\bigotimes_{\substack{p \nmid i \\ 1 \leqq i \leqq n}} B_{i}(e(n, i), e(m, i)$
$+1)$.

We lift the filtration of $C_{n, m}$ defined in the previous section to $\bar{C}_{n, m}$. That is, we define $F_{i} \bar{C}_{n, m}$ to be the $\mathbb{Z}_{(p)}$-submodule spanned by

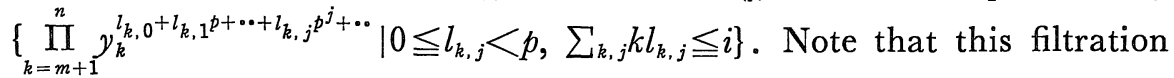
is compatible with the product of $\bar{C}_{n, m}$. We restrict the filtrations of $\bar{C}_{n, m}$ and $C_{n, m}$ to $\bar{B}_{i}(e(n, i), e(m, i)+1)$ and $B_{i}(e(n, i), e(m, i)+1)$, then $F_{k} \bar{B}_{i}(r, s)\left(F_{k} B_{i}(r, s)\right)$ is spanned by

$$
\left\{\prod_{t=s}^{r} a_{i, t}^{l_{t, 0}+l_{t, 1}{ }^{p+\bullet \cdot l_{t, j}} p^{j}+\bullet \bullet} \mid 0 \leqq l_{t, j}<p, \quad \sum_{t, j} i p^{t} l_{t, j} \leqq k\right\}
$$

over $\mathbb{Z}_{(p)}$ (resp. $\left.\mathbb{F}_{p}\right)$. 
To describe the coproduct of $E^{0} B_{i}(r, s)$, we arrange some notations and lemmas.

Notation 3.3. For a non-negative integer s. We define

$$
d_{s, l}(l=0,1,2, \ldots) \text { by } s=d_{s, 0}+d_{s, 1} p+\ldots+d_{s, j} p^{j}+\ldots\left(0 \leqq d_{s, j}<p\right) \text {. }
$$

We put $C(s)=\frac{s !}{(1 !)^{d_{s, 0}}(p !)^{d_{s, 1}} \ldots\left(p^{j !}\right)^{d_{s, j}} .}$,

$$
\operatorname{ord}_{p} s=\max \left\{l \mid d_{s, t}=0 \text { if } t<l\right\} \text {. }
$$

Lemma 3.4. ( i ) For $1 \leqq k \leqq p^{j}, p^{k}\left(\begin{array}{l}p^{j} \\ k\end{array}\right) \equiv 0 \bmod p^{j+1}$ 。

(ii) $\quad C(s) \equiv \prod_{l \geqq 0} d_{s, l} ! \bmod p$.

(iii) For $0<s<p^{j}$,

$$
d_{s, l}+d_{p^{j}-s, l}= \begin{cases}p, & l=\operatorname{ord}_{p} s \\ p-1, & \operatorname{ord}_{p} s<l<j \\ 0, & l<\operatorname{ord}_{p} s \text { or } l \geqq j\end{cases}
$$

(iv) Let $i_{1}, i_{2}, \ldots, i_{p^{j}-1}$ be a sequence of non-negative integers such that $\sum_{s=1}^{p^{j}} i_{s} d_{s, l} \leqq p-1$ and $\sum_{s=1}^{p^{j}-1} i_{s} d_{p^{j}-s, l} \leqq p-1$ for $l=0,1,2, \ldots, j-1$. Then $i_{s}=0$ for all $s$ or there exists $t$ such that $i_{s}=\left\{\begin{array}{l}1, s=t \\ 0, s \neq t .\end{array}\right.$

Proof. (i) It is easy to verify the inequality $\operatorname{ord}_{p} p^{j+k}\left(p^{j}-1\right)\left(p^{j}\right.$ $-2) \ldots\left(p^{j}-(k-1)\right) \geqq j+1+\operatorname{ord}_{p} k$ ! which is equivalent to the assertion.

(ii) Just apply the formula $\left(\begin{array}{l}a \\ b\end{array}\right) \equiv \prod_{l \geq 0}\left(\begin{array}{l}d_{a, l} \\ d_{b, l}\end{array}\right) \bmod p$ to

$$
C(s)=\prod_{l \geq 0} \prod_{i=0}^{d_{s, l^{-1}}}\left(\begin{array}{c}
s-d_{s, 0}-d_{s, 1} p-\ldots-d_{s, l-1} p^{l-1}-i p^{l} \\
p^{l}
\end{array}\right) .
$$

(iii) The $p$-adic expansion of $p^{j}-s$ is $\left(p-d_{s, k}\right) p^{k}+\left(p-1-d_{s, k+1}\right) p^{k+1}$ $+\ldots+\left(p-1-d_{s, j-1}\right) p^{j-1}\left(k=\operatorname{ord}_{p} s\right)$.

(iv) By the assumption, we have $\sum_{s=1}^{p^{j}-1} i_{s}\left(d_{s, l}+d_{p^{j}-s, l}\right) \leqq 2(p-1)$. Applying (iii), we have $\sum_{\text {ord }_{p} s=l} p i_{s}+\sum_{\text {ord }_{p} s<l}(p-1) i_{s} \leqq 2(p-1)$ for $l=0,1$, $2, \ldots, j-1$. Now the result follows easily

Theorem 3.5. Fix $i$ ( $p \nmid i)$ and put $\gamma_{s}=C(s)^{-1} a_{i, 0}^{d_{s, 0}} a_{i, 1}^{d_{s, 1}} \ldots a_{i, j}^{d_{s, j}} \ldots$ $\in \bar{B}_{i}$ for $s=0,1,2, \ldots$, then $\varphi a_{i j} \equiv \sum_{s+t=p^{j}} \gamma_{s} \otimes \gamma_{t}$ modulo $F_{i p^{j}-1}+(p)$ where 
$F_{k}=F_{k}\left(\bar{B}_{i} \otimes \bar{B}_{i}\right)=\sum_{s+t=k} F_{s} \bar{B}_{i} \otimes F_{t} \bar{B}_{i}$ and $(q)$ is an ideal generated by $q$.

Proof. Since $a_{i, 0}=f_{i}$ is primitive, the above assertion is true if $j$ $=0$. Inductively, assume that $\varphi a_{i, k} \equiv \sum_{s+t=p^{k}} \gamma_{s} \otimes \gamma_{t}$ modulo $F_{i p^{k}-1}+(p)$ for $k=0,1, \ldots, j$. Put $\varphi a_{i, k}=\gamma+p \alpha+\beta \quad\left(\gamma=\sum_{s+t=p^{h}} \gamma_{s} \otimes \gamma_{t}, \alpha \in \bar{B}_{i} \otimes \bar{B}_{i}\right.$, $\beta \in F_{i p^{k}-1}$ ). Applying (i) of (3.4), we have

$$
\left(\varphi a_{i, k}\right)^{p^{j+1-k}} \equiv(\gamma+\beta)^{p^{j+1-k}} \bmod \left(p^{j+2-k}\right) .
$$

Since

$$
(\gamma+\beta)^{p^{j+1-k}} \equiv \gamma^{p^{j+1-k}} \bmod F_{i p^{j+1}-1}
$$

we have

$$
\left(\varphi a_{i, k}\right)^{p^{j+1-k}} \equiv\left(\sum_{s+t=p^{h}} \gamma_{s} \otimes \gamma_{t}\right)^{p^{j+1-k}} \bmod F_{i p^{j+1}-1}+\left(p^{j+2-k}\right) \ldots\left(^{*}\right) .
$$

On the other hand,

$$
\begin{aligned}
& \left(\sum_{s+t=p^{h}} \gamma_{s} \otimes \gamma_{t}\right)^{p^{j+1-k}}=\sum_{i_{0}+\cdots+i} p_{p^{k}}=p^{j+1-k} \frac{p^{j+1-k} !}{i_{0} ! \ldots i_{p^{k}} !} \gamma_{1}^{i_{1}} \ldots \gamma_{p^{k}}^{i^{p^{k}}} \otimes \gamma_{1}^{i^{p^{k}}-1} \cdots \gamma_{p^{k}}^{i_{0}} \\
& \equiv \sum_{\substack{i_{0}+\cdots+i \\
i_{s}<p}} p_{p^{k}}=p^{j+1-k} \frac{p^{j+1-k} !}{i_{0} ! \ldots i_{p^{k}} !} \gamma_{1}^{i} \ldots \gamma_{p^{k}}^{i} \otimes \gamma_{1}^{p^{p^{k}}-1} \ldots \gamma_{p^{k}}^{i_{0}} \bmod F_{i p^{j+1}-1} \\
& \equiv\left\{\begin{array}{lr}
0 \bmod \left(p^{j+2-k}\right) & \text { for } k=0,1, \ldots, j-1 \\
\sum_{\substack{i_{0}+\cdots+i \\
i_{s}<p}}=\frac{p !}{i_{0} ! \ldots i_{p^{j}} !} \gamma_{1}^{i_{1}} \cdots \gamma_{p^{j}}^{p^{j}} \otimes \gamma_{1}^{p^{j}-1} \cdots \gamma_{p^{j}}^{i_{0}} \bmod \left(p^{2}\right) & \text { for } k=j .
\end{array}\right.
\end{aligned}
$$

By (*), we have

$$
p^{k}\left(\varphi a_{i, k}\right)^{p^{j+1-k}} \equiv \begin{cases}0, & k=0,1, \ldots j-1 \\ p^{j+1} \sum_{\substack{i_{0}+\cdots+i \\ i_{s}<p}}=p \frac{(p-1) !}{i_{0} ! \ldots i_{p^{j}} !} \gamma_{1}^{i_{1}} \cdots \gamma_{p^{j}}^{i} \otimes \gamma_{1}^{p^{j}}{ }^{i^{j}-1} \cdots \gamma_{p^{j}}^{i_{0}}, \quad k=j\end{cases}
$$

modulo $F_{i p^{j+1}-1}+\left(p^{j+2}\right)$. Apply the coproduct $\varphi$ to the both sides of $a_{i, 0}^{p^{j+1}}+p a_{i, 1}^{p^{j}}+\ldots+p^{j+1} a_{i, j+1}=f_{i p^{j+1}}$. Since $f_{i p^{j+1}}$ is primitive,

$$
\begin{aligned}
& p^{j+1} \varphi a_{i, j+1}+\sum_{k=0}^{j} p^{k}\left(\varphi a_{i, k}\right)^{p^{j+1-k}}=1 \otimes\left(a_{i, 0}^{p^{j+1}}+p a_{i, 1}^{p^{j}}\right. \\
& \left.\quad+. .+p^{j+1} a_{i, j+1}\right)+\left(a_{i, 0}^{j^{j+1}}+p a_{i, 1}^{p^{j}}+\ldots+p^{j+1} a_{i, j+1}\right) \otimes 1 .
\end{aligned}
$$

Therefore we have 


$$
\begin{gathered}
p^{j+1} \varphi a_{i, j+1}+p^{j+1} \sum_{i_{0}+\cdots+i_{p^{j}}=p} \frac{(p-1) !}{i_{0} ! \ldots i_{p^{j}} !} \gamma_{1}^{i_{1}} \cdots \gamma_{p^{j}}^{i_{p^{j}}} \otimes \gamma_{1}^{i_{p^{j}}-1} \cdots \gamma_{p^{j}}^{i_{0}} \\
\equiv 1 \otimes p^{j+1} a_{i, j+1}+p^{j+1} a_{i, j+1} \otimes 1 \text { modulo } F_{i p^{j+1}-1}+\left(p^{j+2}\right) .
\end{gathered}
$$

Since $\bar{B}_{i} \otimes \bar{B}_{i}$ has no $p$-torsion, we have, using the fact $(p-1) ! \equiv-1$ $\bmod p$,

$$
\begin{aligned}
& \varphi a_{i, j+1} \equiv 1 \otimes a_{i, j+1}+a_{i, j+1} \otimes 1
\end{aligned}
$$

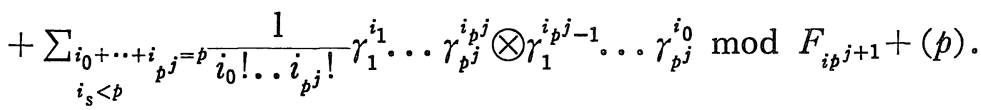

By definition,

$$
\begin{aligned}
& \gamma_{1}^{i_{1}} \cdots \gamma_{p^{j}}^{i_{i}^{j}}=\prod_{s=1}^{p^{j}} C(s)^{-i_{s}} \prod_{l=0}^{j} \gamma_{p^{l}}^{\sum_{s=1}^{p^{j}} s_{s, l} s_{s, l}},
\end{aligned}
$$

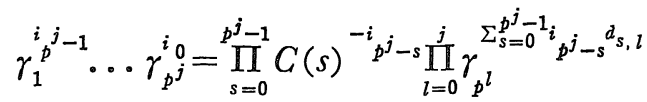

$$
\begin{aligned}
& =\prod_{s=1}^{p^{j}} C\left(p^{j}-s\right)^{-i} \prod_{l=0}^{j} \gamma_{p^{l}}^{\sum_{s=1}^{p^{j}} s^{i} p^{j}-s, l} .
\end{aligned}
$$

Therefore we have

$$
\begin{aligned}
& \varphi a_{i, j+1} \equiv 1 \otimes a_{i, j+1}+a_{i, j+1} \otimes 1+\sum \frac{1}{i_{0} !_{0} . i_{p^{j}} !}\left\{\prod_{s=1}^{p^{j}}\left(C(s) C\left(p^{j}-s\right)\right)^{-i_{s}}\right\}
\end{aligned}
$$

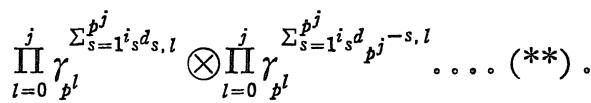

Here the above $\Sigma$ is the summation over $i_{0}, \ldots, i_{p^{j}}$ such that $i_{0} t_{\text {. }}$ $+i_{p^{j}}=p, i_{s}<p$ and $\sum_{s=1}^{p^{j}} i_{s} d_{s, l} \leqq p-1, \sum_{s=1}^{p^{j}} i_{s} d_{p^{j}-s, l} \leqq p-1$ for $l=0,1, \ldots$, $j$. Under this condition,

$$
\begin{aligned}
& \prod_{l=0}^{j} \gamma_{p^{l}}^{\sum_{s=1}^{p^{j}} i^{i} s_{s, l}}=C\left(\sum_{s=1}^{p^{j}} s i_{s}\right) \gamma_{\sum_{s=1}^{p^{j}} s_{s}}
\end{aligned}
$$

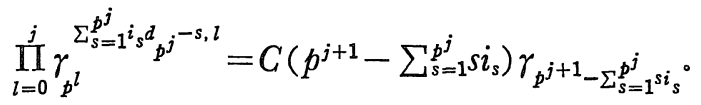

Now apply (iv) of (3.4) to (**),

$$
\begin{aligned}
& \varphi a_{i, j+1}-\left(1 \otimes a_{i, j+1}+a_{i, j+1} \otimes 1\right)
\end{aligned}
$$

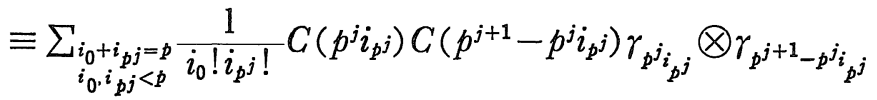

$$
\begin{aligned}
& +\sum_{s=1}^{p^{j}=1} \sum_{i_{0}+i}{ }_{p^{j}}=p^{p-1} \frac{1}{i_{0} ! i_{p^{j}} !}\left(C(s) C\left(p^{j}-s\right)\right)^{-1} C\left(s+p_{p^{j}}^{j} i\right) C\left(p^{j+1}-s-p_{p^{j}}^{j}{ }^{j}\right)
\end{aligned}
$$


$\gamma_{s+p^{j}{ }_{p}^{j}} \otimes \gamma_{p^{j+1}-s-p^{j}{ }_{p^{j}}} \cdot$

By (ii) and (iii) of (3.4), $C\left(p^{j} i_{p^{j}}\right) C\left(p^{j+1}-p^{j} i_{p^{j}}\right) \equiv i_{p^{j}} !\left(p-i_{p^{j}}\right) !, C\left(s+p^{j}\right.$ $\left.i_{p^{j}}\right) C\left(p^{j+1}-s-p^{j} i_{p^{j}}\right) \equiv C(s) C\left(p^{j}-s\right) i_{p^{j}} !\left(p-1-i_{p^{j}}\right)$ ! for $1 \leqq s \leqq p^{j}-1$.

Finally we have

$$
\begin{aligned}
& \varphi a_{i, j+1}-\left(1 \otimes a_{i, j+1}+a_{i, j+1} \otimes 1\right) \\
& \equiv \sum_{t=1}^{p-1} \gamma_{t p^{j}} \otimes \gamma_{(p-t) p^{j}}+\sum_{s=1}^{p^{j}-1} \sum_{t=0}^{p-1} \gamma_{s+t p^{j}} \otimes \gamma_{p^{j+1}-\left(s+t p^{j}\right)}=\sum_{\substack{s, t=t=0^{j+1} \\
s, t \gamma_{s}}} \otimes \gamma_{t}
\end{aligned}
$$

modulo $F_{i p^{j+1}-1}+(p)$. This completes the proof.

Corollary 3.6. Let $\gamma_{j, k} \in E_{i k}^{0} B_{i}(r, 0) \quad\left(0 \leqq k \leqq p^{r+1}-1\right)$ be the class of the $\bmod p$ reduction of $\gamma_{b}^{p^{j}} \in \bar{B}_{i}$. And let $\Gamma_{i}(r, 0, j)$ be the subalgebra of $E^{0} B_{i}(r, 0)$ generated by $\gamma_{j, 1}, \gamma_{j, 2}, \ldots, \gamma_{j, p^{r+1}-1}$, then $\Gamma_{i}(r, 0, j)$ is a Hopf algebra with relations $\gamma_{j, k} \gamma_{j, l}=\left(\begin{array}{c}k+l \\ k\end{array}\right) \gamma_{j, k+l}$ and coproduct $\varphi \gamma_{j, k}=$ $\sum_{l=0}^{k} \gamma_{j, l} \otimes \gamma_{j, k-l} . \quad$ For $0 \leqq s \leqq r$, let $\tilde{\gamma}_{j, l^{l}} \in E^{0} B_{i}(r, s) \quad(0 \leqq l \leqq r-s)$ be the reduction of $\gamma_{j, p^{s+l}} \in E^{0} B_{i}(r, 0)$ by the map $E^{0} B_{i}(r, 0) \rightarrow E^{0} B_{i}(r, s)$. Put $\tilde{\gamma}_{j, k}=C(k)^{-1} \tilde{\gamma}_{j, 1}^{d_{k} 0} \ldots \tilde{\gamma}_{j, p^{r-s}}^{d_{k, r-s}}\left(0 \leqq k \leqq p^{r-s+1}-1\right)$, and let $\Gamma_{i}(r, s, j)$ be the sub Hopf algebra of $E^{0} B_{i}(r, s)$ generated by $\tilde{\gamma}_{j, 1} \tilde{\gamma}_{j, 2}, \ldots, \tilde{\gamma}_{j, p^{r-s+1}-1}$, then $\Gamma_{i}$ $(r, s, j)$ is a Hopf algebra with relations $\tilde{\gamma}_{j, k} \tilde{\gamma}_{j, l}=\left(\begin{array}{c}k+l \\ k\end{array}\right) \tilde{\gamma}_{j, k+l}$ and coproduct $\varphi \tilde{\gamma}_{j, k}=\sum_{l=0}^{k} \tilde{\gamma}_{j, l} \otimes \tilde{\gamma}_{j, k-l}$. And we have the following splittings:

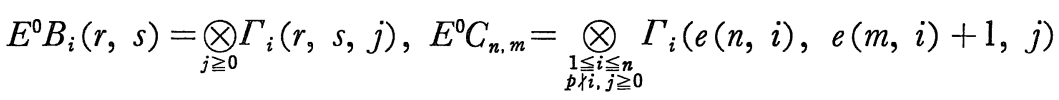

where $\Gamma_{i}(r, r+1, j)=F_{p}$.

Remark 3.7. Let $\alpha$ be a non-zero element of $\mathbb{F}_{p}$. Consider the map $\Gamma_{i}(r, s, j) \rightarrow \Gamma_{i}(r, s, j)$ which sends $\tilde{\gamma}_{j, k}$ to $\alpha^{k} \tilde{\gamma}_{j, k}$. Then, it is an automorphism of Hopf algebra $\Gamma_{i}(r, \mathrm{~s}, j)$. Hence, if we put $\hat{\gamma}_{j, k}$ $=\left\{\frac{(-1)^{i+1}}{i}\right\}^{k} \tilde{\gamma}_{j, k}, \quad\left\{1, \hat{\gamma}_{j, 1}, \ldots, \hat{\gamma}_{j, p^{r+1}-1}\right\}$ spans $\Gamma_{i}(r, s, j)$ :and $\hat{\gamma}_{j, k} \hat{\gamma}_{j, l}=$ $\left(\begin{array}{c}k+l \\ k\end{array}\right) \hat{\gamma}_{j, k+l}, \varphi \hat{\gamma}_{j, k}=\sum_{l=0}^{k} \hat{\gamma}_{j, l} \otimes \hat{\gamma}_{j, k-l}$ hold. Moreover $\hat{\gamma}_{j, p^{l}} \equiv y_{i p^{l+s, j}}$ modulo decomposables in $E^{0} C_{n, m}$, where $\hat{\gamma}_{j, p^{l}} \in \Gamma_{i}(e(n, i), e(m, i)+1, j), s=e(m$, i) +1 .

Lemma 3.8. $h_{i, j}, g_{i, j} \in \operatorname{Cotor}_{*, *}^{E^{0} C_{n, m}}\left(\boldsymbol{F}_{p}, \boldsymbol{F}_{p}\right)\left(i=k p^{s}, p \nmid k, m+1 \leqq i \leqq n\right.$, $s=0$ or $i \leqq m p, j \geqq 0$ ) have representatives $\left[\hat{\gamma}_{j, 1}\right], \sum_{l=1}^{p^{e-s}-1}\left[\hat{\gamma}_{j, l} \mid \hat{\gamma}_{j, p^{e-s}-l}\right]$ in 
$\Omega_{*}\left(E^{0} C_{n, m}\right)$ where $e=e(n, k)$ and $\hat{\gamma}_{j, l} \in \Gamma_{k}(e, s, j)$. (Note that $e(m, k)$ $=s-1$ in this case.)

Proof. This follows from (1.7), (3.6) and (3.7).

Remark 3.9. In $\Omega_{*}\left(E^{0} C_{\infty, m}\right)$, a cycle $\sum_{l=1}^{e^{e-s}-1}\left[\hat{\gamma}_{j, l} \mid \hat{\gamma}_{j, p^{e-s}-l}\right]$ is boun$\operatorname{ded}$ by $\left[\hat{\gamma}_{j, p^{e-s}}\right]$. Hence we denote $\sum_{l=1}^{p^{e-s}-1}\left[\hat{\gamma}_{j, l} \mid \hat{\gamma}_{j, p^{e-s}-l}\right] \in \Omega_{*}\left(E^{0} C_{n, m}\right)$ by $d\left[\hat{\gamma}_{j, p^{e-s}}\right]$. Similarly, although $\left[a_{k, e+1}^{p^{j}}\right] \notin \Omega_{*}\left(C_{n, m}\right), d\left[a_{k, e+1}^{p^{j}}\right]$ is a cycle of $\Omega_{*}\left(C_{n, m}\right)$. (3.8) and this remark imply the following theorem.

Theorem 3.10. $h_{i, j}, g_{i, j} \in \operatorname{Cotor}_{*, *}^{C_{n, m}}\left(\mathbb{F}_{p}, \mathbb{F}_{p}\right) \quad\left(i=k p^{s}, p \nmid k, \quad m+1 \leqq i\right.$ $\leqq n, s=0$ or $i \leqq m p, j \geqq 0)$ are represented by $\frac{(-1)^{k+1}}{k}\left[a_{k, s}^{p^{j}}\right]$ and $\frac{(-1)^{k+1}}{k}$ $d\left[a_{k, e+1}^{p^{j}}\right]$ in $\Omega_{*}\left(C_{n, m}\right)$ respectively, where $e=e(n, k), a_{k, s}^{p^{j}} \in B_{k}(e, s), a_{k, e+1}^{p^{j}}$ $\in B_{k}(e+1, s)$.

Consider the following Bockstein long exact sequence.

$$
\begin{aligned}
& \ldots \rightarrow \operatorname{Cotor}_{s, t}^{\bar{c}_{n, m}}\left(\mathbb{Z}_{(p)}, \mathbb{Z}_{(p)}\right) \stackrel{p \times}{\longrightarrow} \operatorname{Cotor}_{s, t}^{\bar{c}_{n, m}}\left(\mathbb{Z}_{(p)}, \mathbb{Z}_{(p)}\right) \rightarrow \\
& \operatorname{Cotor}_{s, t}^{c_{n, m}}\left(\mathbb{F}_{p}, \mathbb{F}_{p}\right) \rightarrow \text { Cotor }_{s-1, t}^{\bar{c}_{n, m}}\left(\mathbb{Z}_{(p)}, \mathbb{Z}_{(p)}\right) \rightarrow \ldots
\end{aligned}
$$

associated with the short exact sequence $0 \rightarrow \Omega_{*}\left(\bar{C}_{n, m}\right) \stackrel{p \times}{\longrightarrow} \Omega_{*}\left(\bar{C}_{n, m}\right) \rightarrow$ $\Omega_{*}\left(C_{n, m}\right) \rightarrow 0$. Then we have the Bockstein spectral sequence associated with the above long exact sequence.

Theorem 3.11. $\quad h_{i, j} \in \operatorname{Cotor}_{*, *}^{C_{n, m}}\left(\mathbb{F}_{p}, \mathbb{F}_{p}\right)$ is a permanent cycle if $i p^{j} \leqq n$. The differentials of the Bockstein spectral sequence are given by $d^{r} h_{i, j+r}=$ $-g_{i, j}$ for $\left[\frac{n}{p^{r}}\right]<i \leqq\left[\frac{n}{p^{r-1}}\right]$ where we put $g_{i, j}=h_{i, j}^{2}$ if $p=2$ and $i>\left[\frac{n}{2}\right]$.

Proof. Since deg $g_{i, j}=2 i p^{j+e(n, i)+1}-2>2 n-1, h_{i, j}$ is a permanent cycle by dimensional reason, if $i p^{j} \leqq n$.

Note that $r=e(n, i)+1$ if $\left[\frac{n}{p^{r}}\right]<i \leqq\left[\frac{n}{p^{r-1}}\right]$. We put $i=k p^{s}$ where $p \nmid k$, then $i \leqq m p$ if $s>0$ and $r=e(n, k)-s+1$. Recall the defining relation of $a_{i, j}$ 's in $\bar{G}_{\infty, 0 \cdot}$ We have $\sum_{l=0}^{j+r+s} p^{l} a_{k, l}^{p^{j+r+s-l}}=f_{k p^{j+r+s}}$. Apply the reduction $\bar{C}_{\infty, 0} \rightarrow \bar{C}_{\infty, m}$, and we have $\sum_{l=s}^{j+r+s} p^{l} a_{k, l}^{p^{j+r+s-l}}=f_{k p^{j+r+s}}$ in $\bar{C}_{\infty, m}$ since $e(m, k)=s-1$. Hence $f_{k p^{j+r+s}}$ in $\bar{C}_{\infty, m}$ can be divided by $p^{s}$. Therefore 
we have $\left[\sum_{l=0}^{r-1} p^{l} a_{k, s+l}^{p^{j+r-l}}\right]=\left[\frac{1}{p^{s}} f_{k p^{j+r+s}}-p^{r} \sum_{l=0}^{j} p^{l} a_{k, l+r+s}^{p^{j-l}}\right]$ in $\Omega_{-1}\left(\bar{C}_{n, m}\right)$ since the left hand side is contained in $\bar{C}_{n m}$. Noting that $\frac{1}{p^{s}} f_{k p^{j+r+1}}$ is primitive, apply the differential of $\Omega_{*}\left(\bar{C}_{n, m}\right)$ to the both sides of the above equality. Then we obtain $d\left[\sum_{l=0}^{r-1} p^{l} a_{k, s+l}^{p^{j+r-l}}\right] \equiv-p^{r} d\left[a_{k, r+s}^{p^{j}}\right]$ modulo $p^{r+1}$. $\left[\sum_{l=0}^{r-1} p^{l} a_{k, s+l}^{j+r-l}\right] \in \Omega_{-1}\left(\bar{C}_{n, m}\right)$ maps to $\left[a_{k, s}^{j+r}\right] \in \Omega_{-1}\left(C_{n, m}\right)$ which represents $(-1)^{k+1} k h_{i, j}$. Thus we have $d^{r} h_{i, j+r}=-g_{i, j}$.

Remark 3.12. By (3.10), $\operatorname{Cotor}_{*, *}^{B_{k}^{(r, s)}}\left(\boldsymbol{F}_{p}, \boldsymbol{F}_{p}\right)=E\left(h_{k p^{s}, j} \mid j \geqq 0\right) \otimes \boldsymbol{F}_{p}$ $\left[g_{k p^{s}, j} \mid j \geqq 0\right]$ if $p$ is odd or $p=2$ and $r>s$, where bideg $h_{k p^{s}, j}=$ $\left(-1,2 k p^{s+j}\right)$, bideg $g_{k p^{s}, j}=\left(-1,2 k p^{j+r+1}\right)$. The differential of the Bockstein spectral sequence is given by $d^{r-s+1} h_{k p^{s}, j+r-s+1}=-g_{k p^{s}, j^{*}}$ If $p=2, r=s$, then $\operatorname{Cotor}_{*, *}^{B_{k}^{(s, s)}}\left(\boldsymbol{F}_{2}, \boldsymbol{F}_{2}\right)=\boldsymbol{F}_{2}\left[h_{2^{s}, j} \mid j \geqq 0\right]$ and $d^{\mathbb{1}} h_{2^{s} k, j+1}=h_{2^{s} k, j^{\circ}}^{2}$

Corollary 3.13. $h_{i, j}$ and $g_{i, j}$ are primitive.

Proof. Since the homological degree of $h_{i, j}$ is -1 and Cotor ${ }_{0, *}^{C_{n, m}}$ $\left(\boldsymbol{F}_{p}, \boldsymbol{F}_{p}\right)=\boldsymbol{F}_{p}$, it is obvious that $h_{i, j}$ is primitive. In $\operatorname{Cotor}_{*, *}^{\boldsymbol{B}_{k}(r, s)}\left(\boldsymbol{F}_{p}, \boldsymbol{F}_{p}\right)$, $g_{k p^{s}, j}$ is a higher Bockstein image of a primitive element with no inderminacy by (3.12). So $g_{k p^{s}, j} \in \operatorname{Cotor}_{*, *}^{B_{k}(r, s)}\left(\boldsymbol{F}_{p}, \boldsymbol{F}_{p}\right)$ is primitive. The splitting $C_{n, m} \cong \underset{\substack{p+k \\ 1 \leq k \leq n}}{\bigotimes} B_{k}(e(n, k), e(m, k)+1)$ gives an isomorphism

$$
\operatorname{Cotor}_{*, *}^{C_{n, m}}\left(\boldsymbol{F}_{p}, \boldsymbol{F}_{p}\right) \cong \underset{\substack{p k k \\ 1 \leq k \leq n}}{\bigotimes} \operatorname{Cotor}_{*, *}^{B_{k}(e(n, k), e(m, k)+1)}\left(\boldsymbol{F}_{p}, \boldsymbol{F}_{p}\right) .
$$

Hence $g_{i, j} \in \operatorname{Cotor}_{*, *}^{c_{n, m}}\left(\boldsymbol{F}_{p}, \boldsymbol{F}_{p}\right)$ is also primitive.

Theorem 3.14. $\iota_{n, m \sharp}: \operatorname{Cotor}_{*, *}^{C_{n, m}}\left(\boldsymbol{F}_{p}, \boldsymbol{F}_{p}\right) \rightarrow \operatorname{Cotor}_{*, *}^{C_{n+1, m}}\left(\boldsymbol{F}_{p}, \boldsymbol{F}_{p}\right)$ and $\pi_{n, m \neq}: \operatorname{Cotor}_{*, *}^{C_{n, m}}\left(\boldsymbol{F}_{p}, \boldsymbol{F}_{p}\right) \rightarrow \operatorname{Cotor}_{*, *}^{C_{n, m+1}}\left(\mathbb{F}_{p}, \boldsymbol{F}_{p}\right)$ map $h_{i, j}$ and $g_{i, j}$ as stated in (2.6). That is;

$$
\begin{aligned}
\iota_{n, m \sharp}\left(h_{i, j}\right) & =h_{i, j}, \\
\iota_{n, m \sharp}\left(g_{i, j}\right) & = \begin{cases}0, & p \mid n+1, n \geqq m p \text { and } i=k(n, m) \\
g_{i, j}, & \text { therwise }\end{cases} \\
{[} & =\left\{\begin{array}{ll}
0, & 4 \mid n+1, n \geqq 4 m \text { and } i=k(n, m) \\
g_{i, j}, & \text { therwise }
\end{array}\right]
\end{aligned}
$$




$$
\begin{aligned}
& \pi_{n, m_{\#}}\left(h_{i, j}\right)= \begin{cases}0, & i=m+1 \\
h_{i, j}, & i \neq m+1\end{cases} \\
& \pi_{n, m \sharp}\left(g_{i, j}\right)= \begin{cases}g_{i, j,} & i \neq m+1 \\
g_{(m+1) p, j,}, & i=m+1,(m+1) p \leqq n \\
0, & i=m+1,(m+1) p>n\end{cases} \\
& {\left[\begin{array}{ll}
g_{i, j}, & i \neq m+1 \\
g_{2(m+1), j}, & i=m+1, n \geqq 4(m+1) \\
h_{2(m+1), j,}^{2} & i=m+1,2(m+1) \leqq n<4(m+1)
\end{array}\right] .}
\end{aligned}
$$

Proof. By (3.10), $\ell_{n, m \sharp}\left(h_{i, j}\right)=h_{i, j}$ is obvious. Suppose $p \mid n+1$, $n \geqq m p[4 \mid n+1, n \geqq 4 m]$ and $i=k(n, m)$ and put $i=k p^{s}(p \nmid k)$. Then $e(n, k)=e(n+1, k)-1$ and $a_{k, e+1}^{p^{j}} \in C_{n+1, m}$ where $e=e(n, k)$. Hence $\iota_{n, m}\left(g_{i, j}\right)=\frac{(-1)^{k+1}}{k} d\left[a_{k, e+1}^{p^{j}}\right]=0$ in $\operatorname{Cotor}_{*, *}^{C_{n+1, m}}\left(\mathbb{F}_{p}, \mathbb{F}_{p}\right)$. If $i \neq k(n, m)$, an equality $e(n, k)=e(n+1, k)\left(i=k p^{s}, p \nmid k\right)$ holds. Therefore $\ell_{n, m \sharp}\left(g_{i, j}\right)$ $=g_{i, j}$. Note that the condition " $p \mid n+1, n \geqq m p[4 \mid n+1, n \geqq 4 m]$ " equivalent to the condition " $m+1 \leqq k(n, m) \leqq n\left[m+1 \leqq k(n, m) \leqq\left[\frac{n}{2}\right]\right]$, $p \nmid k(n, m)$ or $k(n, m) \leqq m p$ ". $\pi_{n, m}$ maps $B_{k}(e(n, k), e(m, k)+1)$ onto $B_{k}(e(n$, $k), e(m+1, k)+1)$. If $m+1=k p^{s}$ for some $s>0$, ker $\pi_{n, m}$ is an ideal generated by $a_{k, s \cdot}$. And if $p=2,2(m+1) \leqq n<4(m+1)$, then $e(n, k)=$ $s+1$ where $m+1=2^{s} k, 2 \nmid k$. Hence $d\left[a_{k, s+2}^{2^{j}}\right]=\left[a_{k, s+1}^{2^{j}} \mid a_{k, s+1}^{2^{j}}\right]$. These facts imply the assertions on $\pi_{n, m \sharp}$ by $(3.10)$.

\section{§4. Hopf Algebra Structure of $H_{*}\left(\Omega^{2} V_{n, m}\right)$}

Lemma 4.1. The map $H_{k}\left(\Omega^{2} V_{n, m}\right) \rightarrow H_{k}\left(\Omega^{2} V_{\infty, m}\right)$ induced by the inclusion $V_{n, m} \rightarrow V_{\infty, m}$ is an isomorphism for $k \leqq 2 n-1$.

Proof. Since $H_{k}\left(V_{n, m}\right) \rightarrow H_{k}\left(V_{\infty, m}\right)$ is an isomorphism for $k \leqq 2 n+2$, the result follows easily by using the theorem of J. H. C. Whitehead.

Lemma 4.2. The Eilenberg-Moore spectral sequence

$$
E_{s, t}^{2}=\operatorname{Cotor}_{s t}^{C_{\infty, m}}\left(\mathbb{F}_{p,} \mathbb{F}_{p}\right) \Rightarrow H_{s+t}\left(\Omega^{2} V_{\infty, m}\right)
$$

collapses.

Proof. The $E^{2}$-term is generated by $\left\{h_{i, j} \mid i \geqq m+1, p \nmid i\right.$ or $i \leqq m p$, 
$j \geqq 0\}$ which is also a basis of $P E_{*, *}^{2}$. Since the spectral sequence has a structure of a differential Hopf algebra, the above fact implies the assertion.

Corollary 4.3. If $i p^{j} \leqq n, \quad h_{i, j} \in E_{-1,2 i p^{j}}^{2}=\operatorname{Cotor}_{-1.2 i p^{j}}^{c_{n, m}}\left(\mathbb{F}_{p}, \mathbb{F}_{p}\right)$ is a permanent cycle in the Eilenberg-Moore spectral sequence converging to $H^{*}\left(\Omega^{2} V_{n, m}\right)$. In particular, $h_{i, 0}$ is a permanent cycle.

Proof. This follows from (3.14), (4.1) and (4.2).

Corollary 4.4. The sub Hopf algebra of $H_{*}\left(\Omega^{2} V_{n, m}\right)$ generated by $\sum_{k=0}^{2 n-1} H_{k}\left(\Omega^{2} V_{n, m}\right)$ is generated by odd dimensional elements. Hence it is primitively generated.

It is well-known that the homology of $\Omega^{2} V_{i, i-1}\left(=\Omega^{2} S^{2 i+1}\right)$ is given by the following. (See [2] Chapter III, §3, for example.)

$$
H_{*}\left(\Omega^{2} V_{i, i-1}\right)=E\left(h_{i, j} \mid j \geqq 0\right) \otimes F_{p}\left[\beta h_{i, j} \mid j \geqq 1\right] \quad\left[H_{*}\left(\Omega^{2} V_{i, i-1}\right)=\right.
$$
$\left.\mathbb{F}_{2}\left[h_{i, j} \mid j \geqq 0\right]\right]$ where $\operatorname{deg} h_{i, j}=2 i p^{j}-1$ and $\beta$ is the $\bmod p$ Bockstein homomorphism. And the action of the top Dyer-Lashof operation $\xi_{1}$ is given by $\xi_{1} h_{i, j}=h_{i, j+1}$. (4.6) The homology suspension $\sigma_{*}: H_{*}\left(\Omega^{2} V_{i, i-1}\right) \rightarrow H_{*+1}\left(\Omega V_{i, i-1}\right)$ $=C_{i, i-1}$ is given by $\sigma_{*}\left(h_{i, j}\right)=y_{i}^{p^{j}}$.

We need the following property of the Eilenberg-Moore spectral sequence. (See [7] for a proof.)

Proposition 4.7. Let $X$ be a simply connected space. And let $E_{s, t}^{2}=\operatorname{Cotor}_{s, t}^{H_{*}(X)}\left(\mathbb{F}_{p}, \mathbb{F}_{p}\right) \Rightarrow H_{s+t}(\Omega X)$ be the Eilenberg-Moore spectral sequence associated with a path fibration $\Omega X \rightarrow P X \rightarrow X$. Then the following diagram is commutative,

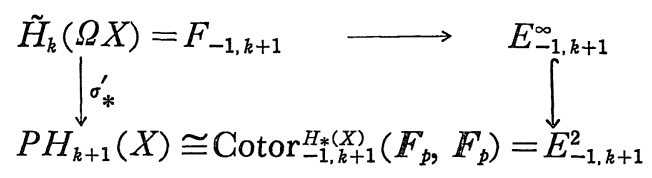

where $\sigma_{*}^{\prime}: \tilde{H}_{k}(\Omega X) \rightarrow P H_{k+1}(X)$ is a map induced by the homology suspension $\sigma_{*}: \tilde{H}_{k}(\Omega X) \rightarrow H_{k+1}(X)$. Hence $\sigma_{*}^{\prime}$ is surjective if and only if $E_{-1, k+1}^{2}=E_{-1, k+1}^{\infty}$. 
Corollary 4.8. In the $E^{2}$-term of the Eilenberg-Moore spectral sequence converging to $H_{*}\left(\Omega^{2} V_{i, i-1}\right), \quad h_{i, j} \in E_{-1,2 i p^{j}}^{2}$ is a permanent cycle which corresponds to $h_{i, j} \in H_{*}\left(\Omega^{2} V_{i, i-1}\right)$.

Proof. Noting that $h_{i, j}=\operatorname{cls}\left[y_{i}^{p^{j}}\right] \in E_{-1,2 i p^{j}}^{2}$, the assertion follows from (4.6) and (4.7).

Lemma 4.9. $h_{i, j} \in E_{-1.2 i p^{j}}^{2}=\operatorname{Cotor}_{-1,2 i p^{j}}^{C_{i, 0}}\left(F_{p}, F_{p}\right)(p \nmid i)$ is a permanent cycle of the Eilenberg-Moore spectral sequence converging to $H_{*}\left(\Omega^{2} V_{i, 0}\right)$. We can choose the unique primitive element $h_{i, j} \in H_{*}\left(\Omega^{2} V_{i, 0}\right)$ corresponding to $h_{i, j}$ in the $E^{2}$-term such that $h_{i, j}=\pi_{i, i-2 *} \circ \circ \circ \circ \pi_{i, 0 *}\left(h_{i, j}\right) \in H_{*}\left(\Omega^{2} V_{i, i-1}\right)$ and $h_{i, j+1}=\xi_{1} h_{i, j}$.

Proof. Corollary (4.4) implies that $P H_{2 i-1}\left(\Omega^{2} V_{i, 0}\right)$ is spanned by a single element because $P\left(\sum_{s+t=2 i-1} E_{s, t}^{2}\right)$ is spanned by a permanent cycle $h_{i, 0}$. Hence we can choose the unique primitive element $h_{i, 0} \in H_{2 i-1}\left(\Omega^{2} V_{i, 0}\right)$ which corresponds to $h_{i, 0}$ in the $E^{2}$-term. Define $h_{i, j} \in H_{*}\left(\Omega^{2} V_{i, 0}\right)$ by $h_{i, j+1}=\xi_{1} h_{i, j}$. It is easy to check that $h_{i, j}=$ $\pi_{i, i-2 *} \circ \cdots \circ \pi_{i, 0 *}\left(h_{i, j}\right)$ holds by applying (3.14) and (4.8). Hence $h_{i, j} \in P H_{2 i p^{j}-1}\left(\Omega^{2} V_{i, 0}\right)$. Since $P\left(\sum_{s+t=2 i p^{j}-1} E_{s, t}^{2}\right)$ is spanned by a single element $h_{i, j} \in E_{-1,2 i j^{j}}^{2}$, it is a permanent cycle and there exists some $\lambda \in F_{p}$ such that $h_{i, j} \in H_{*}\left(\Omega^{2} V_{i, 0}\right)$ corresponds to $\lambda h_{i, j}$ in the $E^{2}$-term. Considering the map between the spectral sequences induced by $\pi_{i, i-2} \circ \circ \circ \pi_{i, 0}: V_{i, 0} \rightarrow V_{i, i-1}$, we see that $\lambda=1$ by $(4.8)$.

Lemma 4. 10. $\quad h_{i p, j} \in E_{-1,2 i p^{j+1}}^{2}=\operatorname{Cotor}_{-1,2 i p^{j+1}}^{c_{i p, i}}\left(\mathbb{F}_{p}, \mathbb{F}_{p}\right)$ is a permanent cycle of the Eilenberg-Moore spectral sequence converging to $H_{*}\left(\Omega^{2} V_{i p, i}\right)$. We can choose the unique primitive element $h_{i p, j} \in H_{*}\left(\Omega^{2} V_{i p, i}\right)$ corresponding to $h_{i p, j}$ in the $E^{2}$-term such that $h_{i p, j}=\pi_{i p, i p-2 *} \circ \circ \circ \circ \pi_{i p, 0 *}\left(h_{i p, j}\right)$ and $h_{i p, j+1}$ $=\xi_{1} h_{i p, j}$.

Proof. Since $P\left(\sum_{s+t=2 i j^{j+1}-1} E_{s, t}^{2}\right)$ is spanned by a single element $h_{i p, j} \in E_{-1,2 i p^{j+1}}^{2}$, the same argument as the above proof works.

Theorem 4.11. The spectral sequence $E_{s, t}^{2}=\operatorname{Cotor}_{s, t}^{C_{n, m}}\left(\mathbb{F}_{p,} \mathbb{F}_{p}\right) \Rightarrow$ 
$H_{s+t}\left(\Omega^{2} V_{n, m}\right)$ collapses.

Proof. By (3.14), $h_{i, j} \in \operatorname{Cotor}_{*, *}^{c_{n, m}}\left(\boldsymbol{F}_{p}, \boldsymbol{F}_{p}\right)$ is the image of $h_{i, j} \in$ Cotor $_{*, *}^{c_{i, 0}}\left(\boldsymbol{F}_{p}, \boldsymbol{F}_{p}\right)$ by $\pi_{n, m-1 \#} \circ \cdots \circ \pi_{n, 0 \#} \circ \ell_{n-1,0 \#} \circ \cdots \circ \ell_{i, 0 \#}$ if $p \nmid i$, and if $p \mid i$, it is the image of $h_{i, j} \in \operatorname{Cotor}_{*, * *}^{C_{i, i / p}}\left(\boldsymbol{F}_{p}, \boldsymbol{F}_{p}\right)$ by $\pi_{n, m-1 \#} \circ \cdots \circ \pi_{n, i / p \#} \circ \ell_{n-1, i / p \#} \circ \cdots \circ \ell_{i, i / p \# *}$ Hence $h_{i, j}$ 's are all permanent cycles by (4.9) and (4.10). Since $g_{i, j}$ 's are all primitive and there is no odd dimensional primitive element in $\sum_{s \leqq-3} E_{s, *}^{2}, g_{i, j}$ s are also permanent cycles.

For any $n, m \geqq 0$, we define $h_{i, j} \in H_{2 i p^{j}-1}\left(\Omega^{2} V_{n, m}\right)$ for $i, j$ such that $m+1 \leqq i \leqq n, p \nmid i$ or $i \leqq m p$ and $j \geqq 0$ by $h_{i, j}=\pi_{n, m-1 *} \circ \cdots \circ \pi_{n, 0 *} \circ \ell_{n-1,0 *} \circ \cdots \circ$ $\iota_{i, 0 *}\left(h_{i, j}\right)$ if $p \nmid i$ where $h_{i, j} \in H_{2 i p^{j}-1}\left(\Omega^{2} V_{i, 0}\right)$ is the element descrived in (4. 9) and $h_{i, j}=\pi_{n, m-1 *} \circ \cdots \circ \pi_{n, i / p *} \circ \ell_{n-1, i / p *} \circ \cdots \circ \ell_{i, i / p *}\left(h_{i, j}\right)$ if $p \mid i$ where $h_{i, j} \in H_{2^{i p^{j}-1}}\left(\Omega^{2} V_{i, i / p}\right)$ is the element described in (4.10).

Lemma 4. 12. If $p=2, h_{i, j}^{2}=0$ in $H_{*}\left(\Omega^{2} V_{n, m}\right)$ for $i \leqq\left[\frac{n}{2}\right]$.

Proof. Suppose that $h_{i, j}^{2} \neq 0$ in $H_{*}\left(\Omega^{2} V_{n, m}\right)$, then $h_{i, j}^{2}$ is a primitive element of degree $2^{j+2} i-2$. On the other hand, since $h_{i, j}^{2}=0$ in the $E^{2}$-term of the Eilenberg-Moore spectral sequence, $h_{i, j}^{2} \in H_{*}\left(\Omega^{2} V_{n, m}\right)$ belongs to $F_{-3, *}$. However, there is no primitive element in $\sum_{\substack{s+t=2^{j+2} \\ s \geqq-3}} E_{i, 2}^{2}$. This contradicts $h_{i, j}^{2} \neq 0$.

Lemma 4. 13. $H_{*}\left(\Omega^{2} V_{n, m}\right)$ is primitively generated.

Proof. By (4.11), $H_{*}\left(\Omega^{2} V_{n, m}\right)$ has generators in degrees $2 i p^{j}-1$ and $2 i p^{j+e(n, i)+1}-2$ for suitable $i, j^{\prime}$ s. Hence if $p$ is an odd prime, there is no indecomposable element in degree $2 k p(k=1,2, \ldots)$. Therefore the assertion is obvious if $p$ is odd. If $p=2$, assume that the square root map $\zeta$ (the dual of squaring map) on $H_{*}\left(\Omega^{2} V_{n, m}\right)$ is non-trivial. Let $x \in H_{*}\left(\Omega^{2} V_{n, m}\right)$ be an element having minimum degree such that $\zeta x \neq 0$, we may assume that $x$ correspond to some $g_{i, j}$ in the $E^{2}$-term of the Eilenberg-Moore spectral sequence. Since $\zeta g_{i, j}=0$ in the $E^{2}$-level, $\zeta x \in F_{-3, *}$. Note that $\zeta$ is a Hopf algebra homomorphism, since $H_{*}\left(\Omega^{2} V_{n, m}\right)$ is cocomutative. Put $\Delta x=1 \otimes x+x \otimes 1$ 
$+\Sigma x^{\prime} \otimes x^{\prime \prime}$ and apply $\zeta$ on the both sides. Then we have $\Delta \zeta x=$ $1 \otimes \zeta x+\zeta x \otimes 1$ by the assumption. Since $\operatorname{deg} x=\operatorname{deg} g_{i, j}=2^{j+e(n, i)+2} i-2 \equiv 2$ $\bmod 4, \zeta x$ is a primitive element of odd degree. But there is no odd dimensional primitive element in $F_{-3, *}$. This contradicts $\zeta x \in F_{-3, *}$. Therefore the square root map on $H_{*}\left(\Omega^{2} V_{n, m}\right)$ is trivial. Thus $H_{*}\left(\Omega^{2} V_{n, m}\right)$ is primitively generated (cf. $[4],(4,20)$ ).

Theorem 4. 14. There are primitive elements $h_{i, j} \in H_{2 i p^{j}-1}\left(\Omega^{2} V_{n, m}\right)$ $(m+1 \leqq i \leqq n, p \nmid i$ or $i \leqq m p, j \geqq 0)$ and $g_{i, j} \in H_{2 i p^{j+e(n, i)+1}-2}\left(\Omega^{2} V_{n, m}\right)(m+1$ $\leqq i \leqq n,\left[m+1 \leqq i \leqq\left[\frac{n}{2}\right]\right.$ if $\left.p=2\right], p \nmid i$ or $\left.i \leqq m p, j \geqq 0\right)$ which satisfy the following:

(i) $h_{i, j}$ and $g_{i, j}$ correspond to $h_{i, j}$ and $g_{i, j}$ in the $E^{2}$-term of the Eilenberg-Moore spectral sequence.

(ii) $\quad H_{*}\left(\Omega^{2} V_{n, m}\right)=E\left(h_{i, j} \mid m+1 \leqq i \leqq n, p \nmid i\right.$ or $\left.i \leqq m p, j \geqq 0\right)$

$\otimes \mathbb{F}_{p}\left[g_{i, j} \mid m+1 \leqq i \leqq n, p \nmid i\right.$ or $\left.i \leqq m p, j \geqq 0\right]$

$\left[H_{*}\left(\Omega^{2} V_{n, m}\right)=E\left(h_{i, j} \mid m+1 \leqq i \leqq\left[\frac{n}{2}\right], 2 \nmid i\right.\right.$ or $\left.i \leqq 2 m, j \geqq 0\right)$

$\otimes \mathbb{F}_{2}\left[g_{i, j} \mid m+1 \leqq i \leqq\left[\frac{n}{2}\right], 2 \nmid i\right.$ or $\left.i \leqq 2 m, j \geqq 0\right]$

$\otimes \mathbb{F}_{2}\left[h_{i, j} \mid \max \left\{\left[\frac{n}{2}\right], m\right\}<i \leqq n, 2 \nmid i\right.$ or $\left.\left.i \leqq 2 m, j \geqq 0\right]\right]$.

(iii) $\iota_{n, m *}\left(h_{i, j}\right)=h_{i, j}$,

$$
\begin{aligned}
& \iota_{n, m *}\left(g_{i, j}\right)=\left\{\begin{array}{l}
0, p \mid n+1, n \geqq m p \text { and } i=k(n, m) \\
g_{i, j}, \text { otherwise }
\end{array}\right. \\
& {\left[= \begin{cases}0, & 4 \mid n+1, \\
g_{i, j}, & \text { otherwise }\end{cases} \right.} \\
& \pi_{n, m *}\left(h_{i, j}\right)= \begin{cases}h_{i, j}, & i \neq m+1 \\
0, & i=m+1\end{cases} \\
& \pi_{n, m *}\left(g_{i, j}\right)= \begin{cases}g_{i, j}, & i \neq m+1 \\
g_{(m+1) p, j}, & i=m+1, n \geqq(m+1) p \\
0, & i=m+1, n<(m+1) p\end{cases} \\
& {\left[\begin{array}{ll}
g_{i, j}, & i \neq m+1 \\
g_{2(m+1), j}, & i=m+1, n \geqq 4(m+1) \\
h_{2(m+1), j}^{2}, & i=m+1,2(m+1) \leqq n<4(m+1)
\end{array}\right] .}
\end{aligned}
$$


(iv) $\xi_{1} h_{i, j}=h_{i, j+1}$.

(v) $h_{i, 0} \in H_{i}\left(\Omega^{2} V_{i, i-1}\right)=H_{i}\left(\Omega^{2} S^{2 i+1}\right)$ is the image of the canonical generator of $H_{2 i-1}\left(S^{2 i-1}\right)$ by the map induced by $S^{2 i-1} \rightarrow \Omega^{2} S^{2 i+1}$.

(vi) $h_{i, j}$ 's and $g_{i, j}$ 's are the unique primitive elements that satisfy the conditions $(\mathrm{i}) \sim(\mathrm{v})$.

Proof. We have already specified the primitive elements $h_{i, j}$ 's. By (4.9) and (4.10), the assertions (iv) and (v) hold. We have $\pi_{n, m *}\left(h_{m+1,0}\right)=0$ by dimensional reason. It follows that $\pi_{n, m *}\left(h_{m+1, j+1}\right)$ $=\pi_{n, m *}\left(\xi_{1} h_{m+1, j}\right)=\xi_{1} \pi_{n, m *}\left(h_{m+1, j}\right)=0$ inductively. Therefore all of the above assertions on $h_{i, j}$ 's hold. Let us consider the Eilenberg-Moore spectral sequence converging to $H_{*}\left(\Omega^{2} V_{i p^{e}, 0}\right)$ where $p \nmid i$ and $e \geqq 0$ $[e \geqq 1$ if $p=2]$. Since $P\left(\sum_{s+t=2 i p^{j+e+1}-2} E_{s, t}^{2}\right)$ is spanned by a single element $g_{i, j}$, there is unique element $g_{i, j}$ in $P H_{2 i p^{j+e+1}-2}\left(\Omega^{2} V_{i p^{e}, 0}\right)$ which corresponds to $g_{i, j}$ in the $E^{2}$-term.

For general $n, m \geqq 0$, we define $g_{i, j} \in H_{2 i p^{j+e(n, i)+1}-2}\left(\Omega^{2} V_{n, m}\right)$ so that the condition (iii) holds. By (3.14), we know that $\iota_{n, 0 *}\left(g_{k(n, 0), j}\right)=0$ modulo filtrations of the Eilenberg-Moore spectral sequence if $p \mid n+1[4 \mid n+1]$; that is, $\iota_{n, 0 *}\left(g_{k(n, 0), j}\right)$ belongs to $P F_{-3,2(n+1) p^{j}+1^{\cdot}}$ But $P E_{s, 2(n+1) j^{j}-2-s}^{2}=0$ if $s \leqq-3$, hence $\iota_{n, 0 *}\left(g_{k(n, 0), j}\right)=0$. The fact that $\iota_{n, m *}\left(g_{k(n, m), j}\right)=0$ if $p \mid n+1, n \geqq m p[4 \mid n+1, n \geqq 4 m]$ for general $m \geqq 0$ follows from the definition of $g_{i, j}$. If $p$ is odd and $n<(m+1) p$, $\pi_{n, m *}\left(g_{m+1, j}\right) \in P F_{-3, *}$ by (3.14). By the same argument as above, we have $\pi_{n, m *}\left(g_{m+1, j}\right)=0$. If $p=2$ and $2(m+1) \leqq n<4(m+1), \pi_{n, m *}\left(g_{m+1, j}\right)$ $-h_{2(m+1), j}^{2} \in P F_{-3, *}$ by (3.14). Similarly we have $\pi_{n, m *}\left(g_{m+1, j}\right)=h_{2(m+1), j}^{2}$. The assertion (ii) is straightforward from (4.11) and (4.12) and the uniqueness is obvious.

Corollary 4. 15. The homology suspension $\sigma_{*}: H_{*}\left(\Omega^{2} V_{n, m}\right) \rightarrow H_{*+1}\left(\Omega V_{n, m}\right)$ maps $h_{i, j}$ to $\frac{(-1)^{k+1}}{k} a_{k, s}^{p^{j}}\left(i=k p^{s}, p \nmid k\right)$ and $\sigma_{*}\left(g_{i, j}\right)=0$.

Proof. This follows from (4.7).

Theorem 4. 16. All of the generators $h_{i, j}, g_{i, j}$ of $H_{*}\left(\Omega^{2} V_{n, m}\right)$ are in the image of the homology suspension $\sigma_{*}: \tilde{H}_{*}\left(\Omega^{3} V_{n, m}\right) \rightarrow H_{*+1}\left(\Omega^{2} V_{n, m}\right)$. 
Proof. $h_{1,0} \in H_{1}\left(\Omega^{2} V_{n, 0}\right)$ is a image of a generator of $H_{1}\left(S^{1}\right)$ by the map induced by $S^{1} \rightarrow \Omega^{2} S^{3} \rightarrow \Omega^{2} S U(n+1)$, and any element of $H_{1}\left(S^{1}\right)$ is in the image of the map induced by $\Sigma \Omega S^{1} \rightarrow S^{1}$ (the adjoint of the identity map of $\Omega S^{1}$ ). Hence $h_{1,0}$ is in the image of the homology suspension. Let $S U(n+1)\langle 3\rangle$ be the three-connective cover of $S U(n+1)$. Then $\Omega^{2} S U(n+1)$ is homotopy equivalent to $\Omega^{2} S U(n+1)\langle 3\rangle \times S^{1}$ and $H_{*}\left(\Omega^{2} S U(n+1)\langle 3\rangle\right)$ is identified with the sub Hopf algebra of $H_{*}\left(\Omega^{2} S U(n+1)\right)$ generated by $h_{i, j}(i \geqq 2$ or $j \geqq 1), g_{i, j}$ We put $\widetilde{V}_{n, m}=\left\{\begin{array}{ll}S U(n+1)\langle 3\rangle, & m=0 \\ V_{n, m}, & m>0\end{array}\right.$. Consider the Eilenberg-Moore spectral sequence associated with the path fibration $\Omega^{3} \widetilde{V}_{n, m} \rightarrow P \Omega^{2} \widetilde{V}_{n, m} \rightarrow$ $\Omega^{2} \widetilde{V}_{n, m}$. By (1.6) and the calculations in $\S 2$, the $E^{2}$-term is given by

$$
\begin{aligned}
& E^{2}=\operatorname{Cotor}^{H_{*}\left(\Omega^{2} \widetilde{V}_{n, m^{\prime}}\right.}\left(\mathbb{F}_{p}, \mathbb{F}_{p}\right)=\mathbb{F}_{p}\left[\tilde{h}_{i, j} \mid m+1 \leqq i \leqq n, i \leqq m p \text { or } p \nmid i,\right. \\
& j \geqq 0(j \geqq 1 \text { if } m=0, i=1)] \otimes E\left(\tilde{g}_{i, j, k} \mid m+1 \leqq i \leqq n, i \leqq m p \text { or } p \nmid i,\right. \\
& j \geqq 0(j \geqq 1 \text { if } m=0, i=1), k \geqq 0) \otimes \mathbb{F}_{p}\left[h_{i, j, k} \mid m+1 \leqq i \leqq n, i \leqq m p \text { or } p \nmid i_{9}\right. \\
& j \geqq 0(j \geqq 1 \text { if } m=0, i=1), k \geqq 0]
\end{aligned}
$$

where

bideg $\tilde{h}_{i, j}=\left(-1,2 \mathrm{i} p^{j}-1\right)$, bideg $\tilde{g}_{i, j, k}=\left(-1,2 p^{k}\left(i p^{j+e(n, i)+1}-1\right)\right)$

bideg $h_{i, j, k}=\left(-2,2 p^{k+1}\left(i p^{j+e(n, i)+1}-1\right)\right)$ and $\tilde{\beta} g_{i, j, k+1}=-h_{i, j, k}$

( $\tilde{\beta}$ is the algebraic Bockstein operator).

$$
\begin{aligned}
& {\left[E^{2}=\text { Cotor }^{H_{+}\left(\Omega^{2} \tilde{V}_{n, m}\right.}\left(\mathbb{F}_{2}, \mathbb{F}_{2}\right)=\mathbb{F}_{2}\left[\tilde{h}_{i, j} \mid m+1 \leqq i \leqq\left[\frac{n}{2}\right], i \leqq 2 m \text { or } 2 \nmid i,\right.\right.} \\
& j \geqq 0 \quad(j \geqq 1 \text { if } m=0, i=1)] \otimes \mathbb{F}_{2}\left[\tilde{g}_{i, j, k} \mid m+1 \leqq i \leqq\left[\frac{n}{2}\right], i \leqq 2 m \text { or } 2 \nmid i\right. \text {, } \\
& j \geqq 0 \quad(j \geqq 1 \text { if } m=0, i=1), k \geqq 0] \otimes \mathbb{F}_{2}\left[\tilde{h}_{i, j, k} \mid \max \left\{\left[\frac{n}{2}\right], m\right\}<i \leqq n,\right. \\
& i \leqq 2 m \text { or } 2 \nmid i, j \geqq 0 \quad(j \geqq 1 \text { if } m=0, i=1), k \geqq 0]
\end{aligned}
$$

where

$$
\begin{aligned}
& \operatorname{bideg} \tilde{h}_{i, j}=\left(-1,2^{j+1} i-1\right) \text {, bideg } \tilde{g}_{i, j, k}=\left(-1,2^{k+1}\left(2^{j+e(n, i)+1} i-1\right)\right) \\
& \text { bideg } \left.\tilde{h}_{i, j, k}=\left(-1,2^{k}\left(2^{j+1} i-1\right)\right)\right] .
\end{aligned}
$$

Note that the Eilenberg-Moore spectral sequence has a structure of Hopf algebra and the above generators $\tilde{h}_{i, j}, \tilde{g}_{i, j, k}, \tilde{h}_{i, j, k}$ are all primitive. Hence, if $p$ is odd, there is no possibility of non-trivial 
differentials by dimensional reason. Therefore the spectral sequence collapses and the assersion follows from (4.7) if $p$ is odd. We consider the case $p=2$. The $E^{2}$-term of the Eilenberg-Moore spectral sequence associated with the fibering $\Omega^{3} \widetilde{V}_{\infty, m} \rightarrow P \Omega^{2} \widetilde{V}_{\infty, m} \rightarrow \Omega^{2} \widetilde{V}_{\infty, m}$ is given by

$$
\begin{aligned}
& E^{2}=\operatorname{Cotor}^{H *\left(\Omega^{2} \tilde{V}_{\infty, m}\right)}\left(\mathbb{F}_{2}, \boldsymbol{F}_{2}\right)=\mathbb{F}_{2}\left[\tilde{h}_{i, j} \mid i \geqq m+1, i \leqq 2 m \text { or } 2 \mid i, j \geqq 0\right. \\
& \left.(j \geqq 1 \text { if } m=0, i=0)] \text { (bideg } \tilde{h}_{i, j}=\left(-1,2^{j+1} i-1\right)\right) .
\end{aligned}
$$

It follows that the spectral sequence collapses and we have $H_{*}\left(\Omega^{3} \widetilde{V}_{\infty, m}\right)$ $\cong \mathbb{F}_{2}\left[\tilde{h}_{i, j} \mid i \geqq m+1, i \leqq 2 m\right.$ or $2 \nmid i, j \geqq 0 \quad(j \geqq 1$ if $\left.m=0, i=1)\right]$.

By (4.7), $\sigma_{*}: H_{*}\left(\Omega^{3} \widetilde{V}_{\infty, m}\right) \rightarrow H_{*+1}\left(\Omega^{2} \widetilde{V}_{\infty, m}\right)$ maps $\tilde{h}_{i, j}$ to $h_{i, j}$. Since the maps $H_{t}\left(\Omega^{3} \widetilde{V}_{n, m}\right) \rightarrow H_{t}\left(\Omega^{3} \widetilde{V}_{\infty, m}\right)$ and $H_{t+1}\left(\Omega^{2} \widetilde{V}_{n, m}\right) \rightarrow H_{t+1}\left(\Omega^{2} \widetilde{V}_{\infty, m}\right)$ are bijective for $t \leqq 2 n-2, h_{i, 0} \in H_{*}\left(\Omega^{2} \widetilde{V}_{n, m}\right)$ is in the image of $\sigma_{*}$. The commutativity of $\sigma_{*}$ with homology operations ([2], Theorem 1.4) implies that $h_{i, j} \in H_{*}\left(\Omega^{2} \widetilde{V}_{n, m}\right)$ is in the image of $\sigma_{*}$ and that $h_{i, j}^{2^{k}} \in H_{*}$ $\left(\Omega^{2} \widetilde{V}_{n, m}\right)$ is also in the image of $\sigma_{*}$. Therefore $\tilde{h}_{i, j}$, and $\tilde{h}_{i, j, k}$ are permanent cycles in the Eilenberg-Moore spectral sequence converging to $H_{*}\left(\Omega^{2} \widetilde{V}_{n, m}\right)$ by (4.7). On the other hand, $\tilde{g}_{i, j, k}$ 's are permanent cycle by dimensional reason. Thus the Eilenberg-Moore spectral sequence collapses and we have the result applying (4.7).

Corollary 4.17. There are the following isomorphisms as algebras. $H_{*}\left(\Omega^{3} \widetilde{V}_{n, m}\right) \cong \tilde{F}_{p}\left[\tilde{h}_{i, j} \mid m+1 \leqq i \leqq n, i \leqq m p\right.$ or $p \nmid i, j \geqq 0 \quad(j \geqq 1 \quad$ if $m=0$, $i=1)] \otimes E\left(\tilde{g}_{i, j, k} \mid m+1 \leqq i \leqq n, i \leqq m p \quad\right.$ or $p \nmid i, j \geqq 0 \quad(j \geqq 1$ if $m=0, i=1)$, $k \geqq 0) \otimes \boldsymbol{F}_{p}\left[h_{i, j, k} \mid m+1 \leqq i \leqq n, i \leqq m p \quad\right.$ or $p \nmid i, j \geqq 0 \quad(j \geqq 1$ if $m=0, i=1)$, $k \geqq 0]$

$$
\begin{aligned}
& \quad\left[H _ { * } ( \Omega ^ { 3 } \widetilde { V } _ { n , m } ) \cong \mathbb { F } _ { 2 } \left[\tilde{h}_{i, j} \mid m+1 \leqq i \leqq\left[\frac{n}{2}\right], i \leqq 2 m \text { or } 2 \nmid i, j \geqq 0(j \geqq 1 \text { if } m=0,\right.\right. \\
& i=1)] \otimes \boldsymbol{F}_{2}\left[\tilde{g}_{i, j, k} \mid m+1 \leqq i \leqq\left[\frac{n}{2}\right], i \leqq 2 m \text { or } 2 \nmid i, j \geqq 0 \quad(j \geqq 1 \text { if } m=0,\right. \\
& i=1), k \geqq 0] \otimes \boldsymbol{F}_{2}\left[\tilde{h}_{i, j, k} \mid \max \left\{\left[\frac{n}{2}\right], m\right\}<i \leqq n, i \leqq 2 m \text { or } 2 \nmid i, j \geqq 0 \quad(j \geqq 1 \text { if }\right. \\
& m=0, i=1), k \geqq 0]] \\
& \text { where } \quad \operatorname{deg} \tilde{h}_{i, j}=2 i p^{j}-2, \operatorname{beg} \tilde{g}_{i, j, k}=2 p^{k}\left(i p^{j+e(n, i)+1}-1\right)-1, \\
& \quad \operatorname{deg} h_{i, j, k}=2 p^{k+1}\left(i p^{j+e(n, i)+1}-1\right)-2, \operatorname{deg} \tilde{h}_{i, j, k}=2^{k}\left(2^{j+1} i-1\right)-1 .
\end{aligned}
$$

\section{§5. Bockstein Spectral Sequence of $H_{*}\left(\Omega^{2} V_{n, m}\right)$}

In order to apply (3.11) to calculation of the Bockstein spectral 
sequence of $H_{*}\left(\Omega^{2} V_{n, m}\right)$, we need the following fact (see [7] for a proof).

Theorem 5.1. Let $\begin{gathered}E^{\prime} \rightarrow E \\ \downarrow \\ B^{\prime} \rightarrow B\end{gathered}$ be a fiber square such that the following conditions are satisfied;

(i) $B$ is simply connected.

(ii) $E \rightarrow B$ is a Serre fibering

(iii) $H_{*}\left(B ; \mathbb{Z}_{(p)}\right), H_{*}\left(E ; \mathbb{Z}_{(p)}\right)$ and $H_{*}\left(B^{\prime} ; \mathbb{Z}_{(p)}\right)$ are torsion free. Let $\left\{E_{s, t}^{r}, d^{r}\right\}$ be the Eilenberg-Moore spectral sequence associated with the fiber square in the mod $p$ homology and let $\left\{\bar{E}_{s, t}^{r}, \bar{d}^{r}\right\}$ be the Eilenberg-Moore spectral sequence associated with the fiber square in the homology of $\mathbb{Z}_{(p)}{ }^{-}$ coefficients. If $y \in E_{s, t}^{2}$ is a permanent cycle, $\tilde{\delta} y \in \bar{E}_{s-1, t}^{2}$ is also a permanent cycle where

$$
\begin{aligned}
& \tilde{\delta}: E_{s, t}^{2}=\operatorname{Cotor}_{s, t}^{H_{\Upsilon}\left(B ; F_{p}\right)}\left(H_{*}\left(B^{\prime} ; \mathbb{F}_{p}\right), H_{*}\left(E ; \mathbb{F}_{p}^{\prime}\right)\right) \rightarrow \\
& \bar{E}_{s-1, t}^{2}=\operatorname{Cotor}_{s-1, t}^{H_{*}\left(B ; Z_{(p)}\right)}\left(H_{*}\left(B^{\prime} ; \mathbb{Z}_{(p)}\right), H_{*}\left(E ; \mathbb{Z}_{(p)}\right)\right)
\end{aligned}
$$

is the algebraic Bockstein homomorphism. Let $\tilde{y} \in F_{s, t}$ be the element corresponding to $y \in E_{s, t}^{2}$, then $\delta \bar{y} \in \bar{F}_{s-1, t}$ and $\delta \bar{y}$ corresponds to the permanent cycle - $\tilde{\delta} y$, where $\delta: H_{*}\left(E^{\prime} ; \mathbb{F}_{p}\right) \rightarrow H_{*-1}\left(E^{\prime} ; \mathbb{Z}_{(p)}\right)$ is the geometric Bockstein homomorphism.

We apply the above theorem to a fiber square

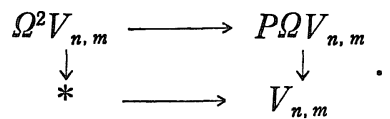

Theorems (3.11) and (4.14) yield the following.

Lemma 5. 2. $\delta h_{i, j} \in H_{2 i p^{j}-2}\left(\Omega^{2} V_{n, m} ; \mathbb{Z}_{(p)}\right)$ can not be divided by $p^{e(n, i)+1}$ if $i p^{j}>n$. Hence $d^{s} h_{i, j} \neq 0$ for some $s \leqq e(n, i)+1$ in the Bockstein spectral sequence of $H_{*}\left(\Omega^{2} V_{n, m}\right)$ if $i p^{j}>n$.

Proof. By (3.11), the algebraic Bockstein homomorphism $\tilde{\delta}$ sends $h_{i, j}$ to an element which can not be divided by $p^{e(n, i)+1}$.

Since $\tilde{\delta} h_{i, j} \in \bar{E}_{-2,2 i p^{j}}^{2}$ and $\bar{E}_{0, *}^{2} \cong Z_{(p)}$ in the Eilenberg-Moore spectral sequence converging to $H_{*}\left(\Omega^{2} V_{n, m} ; \mathbb{Z}_{(p)}\right), \tilde{\delta} h_{i, j}$ is not bounded and repre- 
sents a non-trivial element of $H_{*}\left(\Omega^{2} V_{n, m} ; \mathbb{Z}_{(p)}\right)$. Hence $\delta h_{i, j} \in \bar{F}_{-2, *}$ $-\bar{F}_{-3, *}$ and $\delta h_{i, j}$ corresponds to $-\tilde{\delta} h_{i, j}$ by (5.1). Thus $d^{s} h_{i, j} \neq 0$ for some $s$. Then we may put $\delta h_{i, j}=p^{s-1} \gamma$ for some $\gamma$ such that $\gamma$ is not divided by $p$. Consider the reduction of $\delta h_{i, j}=p^{s-1} \gamma$ to the $\bar{E}^{2}$-term. We see that $\tilde{\delta} h_{i, j}$ is divided by $p^{s-1}$. This implies $s-1<e(n, i)+1$, that is, $s \leqq e(n, i)+1$.

Lemma 5.3. In $H_{*}\left(\Omega^{2} V_{i, i-1}\right)$, the action of the Bockstein homomorphism $\beta$ is given by $\beta h_{i, 0}=0, \beta h_{i, j+1}=g_{i, j}$, where $h_{i, j}$ and $g_{i, j}$ are elements specified in (4.14). [If $p=2$, we put $g_{i, j}=h_{i, j}^{2}$ ]

Proof. $\beta h_{i, 0}=0$ is obvious. Since $\delta h_{i, j+1} \in H_{*}\left(\Omega^{2} V_{i, i-1}: \mathbb{Z}_{(p)}\right)$ is represented by $-\tilde{\delta} h_{i, j+1} \in E_{-2,2 i p^{j+1}}^{2}$ in the Eilenberg-Moore spectral sequence of $\mathbb{Z}_{(p)}$-homology and the $\bmod p$ reduction of $-\delta h_{i, j+1}$ is $g_{i, j} \in E_{-2,2 i p^{j+1}}^{2}$ by (3.11), it follows that $\beta h_{i, j+1} \in H_{*}\left(\Omega^{2} V_{i, i-1}\right)$ is represented by $g_{i, j} \in E_{-2,2 i p^{j+1}}^{2}$ in the Eilenberg-Moore spectral sequence. $\beta h_{i, j+1}$ is a non-zero primitive element and we may put $\beta h_{i, j+1}=\lambda g_{i, j}\left(\lambda \in \mathbb{F}_{p}\right)$. By the above argument, we have $\lambda=1$.

Lemma 5. 4. In $H_{*}\left(\Omega^{2} V_{n, m}\right), \beta h_{i, j+1}=g_{i, j}$ if $e(n, i)=0, \beta h_{i, j+1}=0$ if $e(n, i)>0$. [We put $g_{i, j}=h_{i, j}^{2}$ if $p=2$ and $e(n, i)=0$.]

Proof. First we show that $\beta h_{i, j+1}=g_{i, j}$ in $H_{*}\left(\Omega^{2} V_{i, 0}\right)$ if $p \nmid i$ and $\beta h_{i, j+1}=g_{i, j}$ in $H_{*}\left(\Omega^{2} V_{i, i / p}\right)$ if $p \mid i$. Since $\operatorname{dim} P H_{2 i p^{j+1}-2}\left(\Omega^{2} V_{i, 0}\right)=$ $\operatorname{dim} P H_{2 i p^{j+1}-2}\left(\Omega^{2} V_{i, i / p}\right)=1$, we may put $\beta h_{i, j+1}=\lambda g_{i, j}\left(\lambda \in F_{p}\right)$ in each case. Considering the maps induced by $V_{i, 0} \rightarrow V_{i, i-1}, V_{i, i / p} \rightarrow V_{i, i-1}$, we have $\lambda=1$ by (4.14) and (5.3). By (4.14),

$$
\pi_{n, m-1 *} \circ \cdots \circ \pi_{n, 0_{*}} \circ \ell_{n-1,0 *} \circ \cdots \circ \ell_{i, 0_{*}}\left(g_{i, j}\right)=\left\{\begin{array}{l}
g_{i, j}, e(n, i)=0,0 \leqq m<i \\
0, \text { otherwize }
\end{array}\right.
$$

and

$$
\pi_{n, m-1_{*}} \circ \cdots \circ \pi_{n, 0 *} \circ \ell_{n-1,0_{*}} \circ \cdots \circ \ell_{i, 0_{*}}\left(h_{i, j}\right)= \begin{cases}h_{i, j}, & 0 \leqq m<i \\ 0, & i \leqq m\end{cases}
$$

It follows that

$$
\beta h_{i, j+1}=\left\{\begin{array}{ll}
g_{i, j}, & e(n, i)=0 \\
0, & e(n, i)>0
\end{array} \text { in } H_{*}\left(\Omega^{2} V_{n, m}\right) \text { if } p \nmid i .\right.
$$


Similarly we have

$$
\beta h_{i, j+1}=\left\{\begin{array}{ll}
g_{i, j}, & e(n, i)=0 \\
0, & e(n, i)=1
\end{array} \text { in } H_{*}\left(\Omega^{2} V_{n, m}\right) \text { if } p \mid i\right.
$$

Theorem 5.5. The differentials of the Bockstein spectral sequence of $H_{*}\left(\Omega^{2} V_{n, m}\right)$ are given by $d^{e(n, i)+1} h_{i, j+e(n, i)+1}=g_{i, j}$ and $h_{i, j}$ is a permanent cycle if $i p^{j} \leqq n$ [We put $g_{i, j}=h_{i, j}^{2}$ if $p=2$ and $\left.e(n, i)=0\right]$.

Proof. Since the Bockstein spectral sequence has a structure of a differential Hopf algebra, it follows that $h_{i, j}\left(i p^{j} \leqq n\right)$ and $g_{i, j}$ (any $i, j$ ) are permanent cycles by dimensional reason. We assume inductively that $d^{e(n, i)+1} h_{i, j+e(n, i)+1}=g_{i, j}$ if $e(n, i)+1<r$ and that $d^{s} h_{i, j+e(n, i)+1}=0$ if $e(n, i)+1 \geqq r$ and $1 \leqq s<r$. Note that the first assumption implies that $d^{s} h_{i, j+e(n, i)+1}=0$ if $s<e(n, i)+1<r$. By the preceding lemma, the assumptions are true when $r=2$. Under the assumptions, the $E^{r}$-term of the Bockstein spectral sequence becomes

$$
\begin{aligned}
& E^{r}=E\left(h_{i, j} \mid i p^{j} \leqq n\right) \bigotimes E\left(h_{i, j+e(n, i)+1} \mid e(n, i) \geqq r-1, j \geqq 0\right) \\
& \otimes \mathbb{F}_{p}\left[g_{i, j} \mid e(n, i) \geqq r-1, j \geqq 0\right] .
\end{aligned}
$$

For each $i$ such that $e(n, i)=r-1, d^{r} h_{i, j+r} \neq 0$ by the second assumption and (5.2). Since $d^{r} h_{i, j+r}$ is a primitive element of degree $2 i p^{j+r}-2$, we may put $d^{r} h_{i, j+r}=\lambda g_{i, j}\left(\lambda \in \mathbb{F}_{p}\right)$. This implies that $\delta h_{i, j+r}=p^{r-1} \gamma$ for some $\gamma \in H_{2 i p^{j+r}-2}\left(\Omega^{2} V_{n, m}: Z_{(p)}\right)$ and the $\bmod p$ reduction of $\gamma$ is $\lambda g_{i, j \circ}$ Let $\tilde{\gamma}$ be the permanent cycle corresponding to $\gamma$ in the EilenbergMoore spectral sequence. Then we have $\tilde{\delta} h_{i, j+r}=-p^{r-1} \tilde{\gamma}$ by (5.1). It follows that the $\bmod p$ reduction of $\tilde{\gamma}$ is $g_{i, j}$ in the $E^{2}$-term by (3.11). This implies that $\lambda=1$. Then apply $\iota_{n-1, m *} \circ \circ \circ \circ \ell_{i p^{r-1}, m *}$ on the both sides of $d^{r} h_{i, j+r}=g_{i, j}$ where $h_{i, j+r}, g_{i, j} \in H_{*}\left(\Omega^{2} V_{i p^{r-1}, m}\right)$ and $n \geqq i p^{r-1}$. By (4.11) we have

$$
d^{r} h_{i, j+r}=\left\{\begin{array}{ll}
g_{i, j} & \text { if } e(n, i)=r-1 \\
0 & \text { if } e(n, i)>r-1
\end{array} \text { in } H_{*}\left(\Omega^{2} V_{n, m}\right) .\right.
$$

This completes the induction.

\section{§6. Steenrod Action on $H_{*}\left(\Omega^{2} V_{n, m}\right)$ 。}

Throughout this section, we denote the $2 i$-th Steenrod square $S q^{2 i}$ by $P^{i}$ when a prime $p$ is 2 . 
Lemma 6.1. The action of the Steenrod operation on $H_{*}(\Omega S U)=$ $\mathbb{F}_{p}\left[y_{1}, y_{2}, \ldots, y_{i}, ..\right]$ is given by $P_{*}^{i} y_{k}=\left(\begin{array}{c}k-i(\underset{i}{p}-1) \\ k\end{array}\right) y_{k-i(p-1)}$.

Proof. Since $P^{i} x^{k}=\left(\begin{array}{c}k \\ i\end{array}\right) x^{k+i(p-1)}$ in $H^{*}\left(G P^{\infty}\right)=\mathbb{F}_{p}[x]$, we have $P^{i} \gamma_{k}$ $=\left(\begin{array}{c}k-i(p-1) \\ i\end{array}\right) \gamma_{k-i(p-1)}$ in $H_{*}\left(C P^{\infty}\right)=\Gamma(\gamma)$ where $\gamma_{k}$ is the dual of $x^{k}$. $y_{i} \in H_{2 i}(\Omega S U)$ is the image of $\gamma_{i} \in H_{2 i}\left(C P^{\infty}\right)$ by the map induced by $C P^{\infty} \rightarrow \Omega S U$ which is the adjoint of the canonical inclusion $\Sigma C P^{\infty} \rightarrow$ $S U$. So we have the result.

Corollary 6.2. The Steenrod operation acts on the $i$-th Newton polynomial $f_{i} \in H_{*}(\Omega S U) \quad(p \nmid i)$ as follows

$$
\begin{aligned}
& P_{*}^{1} f_{i}=i f_{i-p+1}, P_{*}^{p^{k}} f_{i}=\left(d_{i, k}+1\right) f_{i-p^{k}(p-1)} \text { for } k \geqq 1 \text { where } \\
& f_{i}=0 \text { if } i \leqq 0 .
\end{aligned}
$$

Proof. Suppose $i \not \equiv-1 \bmod p$. Since $P_{*}^{1} f_{i}$ is primitive and $P H_{2 i}(S U)$ is spanned by a single element $f_{k}$, we may put $P_{*}^{1} f_{i}=\lambda f_{i-p+1}$. Note that $f_{i} \equiv(-1)^{i+1} i y_{i}, f_{i-p+1} \equiv(-1)^{i+1}(i+1) y_{i-p+1}$ modulo decomposables and that $P_{*}^{1}$ maps decomposable elements to decomposable elements. Hence $P_{*}^{1} f_{i} \equiv(-1)^{i+1} i(i+1) y_{i-p+1}$ modulo decomposables. Thus $\lambda=i$ and we have $P_{*}^{1} f_{i}=i f_{i-p+1}$ if $i \not \equiv-1 \bmod p$. Note that the formula $P_{*}^{1} f_{i}=i f_{i-p+1}$ is valid if $p \mid i$ since $P_{*}^{1} f_{k p}=P_{*}^{1} f_{k}^{b}=0$. We may put $P_{*}^{1} f_{k p-1}=\lambda_{k} f_{(k-1) p}$ as above. Applying $P_{*}^{1}$ on the both sides of $f_{k p-1}=\sum_{s=1}^{k p-2}(-1)^{s+1} y_{s} f_{k p-1-s}+(-1)^{k+1} y_{k p-1}$, we have

$$
\begin{aligned}
\lambda_{k} f_{(k-1) p} & =\sum_{s=1}^{k p-2}(-1)^{s+1}\left\{(s+1) y_{s-p+1} f_{k p-1-s}+y_{s} P_{*}^{1} f_{k p-1-s}\right\} \\
& =\sum_{\substack{1 \leq s \leq k-1) p-1 \\
p \nmid s}}(-1)^{s} y_{s} f_{(k-1) p-s}+\sum_{s=1}^{k-2}(-1)^{s+1} \lambda_{k-s} y_{s p} f_{(k-s-1) p} \\
& =\sum_{s=1}^{p k-1) p-1}(-1)^{s} y_{s} f_{(k-1) p-s}+\sum_{s=1}^{k-2}(-1)^{s+1}\left(\lambda_{k-s}+1\right) y_{s p} f_{(k-s-1) p} \\
& =-f_{(k-1) p}+\sum_{s=1}^{k-2}(-1)^{s+1}\left(\lambda_{k-s}+1\right) y_{s p} f_{(k-s-1) p \cdot}
\end{aligned}
$$

Therefore $\left(\lambda_{k}+1\right) f_{(k-1) p}=\sum_{s=1}^{k-2}(-1)^{s+1}\left(\lambda_{k-s}+1\right) y_{s p} f_{(k-s-1) p}$ for $k=2$, $3, \ldots$ Hence $\lambda_{k}=-1$ and $P_{*}^{1} f_{k p-1}=-f_{(k-1) p}$. We put $P_{*}^{p^{k}} f_{i}=\lambda f_{i-p^{k}(p-1)}$ as usual. Comparing the coefficients of $y_{i-p^{k}(p-1)}$, we have $\lambda=$ $\left(\begin{array}{c}i-p^{k}(p-1) \\ p^{k}\end{array}\right)=d_{i, k}+1$.

Lemma 6.3. The Steenrod operation acts on $a_{k, s} \in H_{*}\left(\Omega V_{k p^{s}, k p^{s-1}}\right)$ $=\bigotimes_{i} B_{i}\left(e\left(k p^{s}, i\right), e\left(k p^{s-1}, i\right)+1\right)(p \nmid k)$ as follows. $1 \leq i \leq k p^{s}$ 


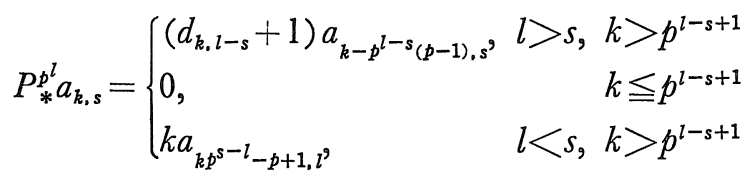

$$
\begin{aligned}
& P_{*}^{p^{s}} a_{k, s}= \begin{cases}k a_{k-p+1, s}, & k>p, p \nmid k+1 \\
0, & k \leqq p \text { or } p \mid k+1\end{cases}
\end{aligned}
$$

Proof. We know that $P H_{*}\left(\Omega V_{k p^{s}, k p^{s-1}}\right)$ is spanned by $a_{i, \ell\left(k p^{\left.p^{s-1}, i\right)+1}\right.}^{p^{j}}$ $\left(1 \leqq i \leqq k p^{s}, p \nmid i, j \geqq 0\right)$. So $P_{*}^{p^{l}} a_{k, s}$ is a linear combination of such elements. Suppose deg $P_{*}^{p^{l}} a_{k, s}=\operatorname{deg} a_{\imath, e+1}^{p^{j}}$ where $e=e\left(k p^{s-1}, i\right)$, then we have $k p^{s}-p^{l}(p-1)=i p^{j+e+1}$. Hence $k p^{s-1}=i p^{j+e}+p^{l-1}(p-1)>i p^{j+e}$ which means $e \geqq j+e$. Thus $j=0$ and $k p^{s}-p^{l}(p-1)=i p^{e+1} \ldots$ (*) $^{*}$

The case $l>s$ : By $\left(^{*}\right), k-p^{l-s}(p-1)=i p^{e+1-s}$. Since $p \nmid k$ we have $e=s-1$ and $i=k-p^{l-s}(p-1)$. The fact $e=s-1$ implies $i p^{s}>k p^{s-1}$; that is, $k>p^{l-s+1}$. Therefore $P_{*}^{p^{l}} a_{k, s}=0$ if $k \leqq p^{l-s+1}$. If $k>p^{l-s+1}$, we may put $P_{*}^{p^{l}} a_{k, s}=\lambda a_{k-p^{l-s}(p-1), s^{*}} \quad$ Since $a_{k, s} \equiv(-1)^{k+1} k y_{k p^{s},} a_{k-p^{l-s}(p-1), s} \equiv$ $(-1)^{k+1} k y_{k p^{s}-p^{l}(p-1)}$ modulo decomposables, comparing the coefficients of $y_{k p^{s}-p^{l}(p-1)}$, we have the result.

The case $l<s:$ By $\left(^{*}\right), k p^{s-l}-p+1=i p^{e+1-l}$. The same argument as above implies $i=k p^{s-l}-p+1, k>p^{l-s+1}$, comparing the coefficients of $y_{k p^{s}-p^{l}(p-1)}$, the result follows.

The case $l=s, p \nmid k+1$ : It follows from $(*)$ that $i=k-p+1$ and $k>p$. We have the result in the same way.

The case $l=s, p \mid k+1$ : We put $k=r p-1$. Then $\left(^{*}\right)$ yields $r=1+$ $i p^{e-s}$. Suppose such $i$ exists and we put $P_{*}^{p^{s}} a_{k, s}=\lambda a_{i, e+1}$. Comparing the coefficients of $y_{k p^{s}-p^{s}(p-1)}$, we have $\lambda=0$

Theorem 6.4. The action of $P_{*}^{p^{k}}$ on $h_{i p^{t}, j} \in H_{*}\left(\Omega^{2} V_{n, m}\right)(p \nmid i, m+1$ $\leqq i p^{t} \leqq n, t=0$ or $\left.i p^{t} \leqq m p, j \geqq 0\right)$ is given as follows.

(i) The case $t=0$ :

$$
P_{*}^{p^{k}} h_{i, j}= \begin{cases}\left(d_{i, k-j}+1\right) h_{i-p^{k-j}(p-1), j}, & k>j, i-p^{k-j}(p-1) \geqq m+1 \\ (i+1) h_{i-p+1, j,} & k=j, p \nmid i+1, i-p \geqq m \\ l h_{l, j+s}, & k=j, i-p+1=l p^{s}, s \geqq 1, \\ & \multicolumn{1}{c}{\quad p \nmid l, l \geqq m+1} \\ 0, & \text { otherwiser }\end{cases}
$$


(ii) The case $t>0$ :

$$
P_{*}^{p^{k}} h_{i p^{t}, j}= \begin{cases}\left(d_{i, k-j-t}+1\right) h_{i p^{t}-p^{k-j}(p-1), j}, & k \geqq j+t, i>p^{k-j-t+1}, \\ & i p^{t}-p^{k-j}(p-1) \geqq m+1 \\ h_{i p^{t}-p^{k-j}(p-1), j}, & j \leqq k<j+t, i>p^{k-j-t+1}, \\ 0, & i p^{t}-p^{k-j}(p-1) \geqq m+1 \\ & \text { otherwise }\end{cases}
$$

Proof. By (4. 14), each $h_{i p^{t}, j}$ is the image of $h_{i, j} \in H_{*}\left(\Omega^{2} V_{i, 0}\right)$ if $t=0$ and the image of $h_{i p^{t}, j} \in H_{*}\left(\Omega^{2} V_{i p^{t}, i p^{t-1}}\right)$ if $t>0$. And we know that $\iota_{n, m *}$ maps the subspace spanned by $h_{i, j}$ 's injectively and that $\operatorname{ker} \pi_{n, m *} \cap$ (the subspace spanned by $h_{i, j}$ 's) is spanned by $h_{m+1, j}(j=$ $0,1,2, \ldots)$. Hence it suffices to examine the action of $P_{*}^{p^{k}}$ on $h_{i, j} \in$ $H_{*}\left(\Omega^{2} V_{i, 0}\right)$ and $h_{i p^{t}, j} \in H_{*}\left(\Omega^{2} V_{i p^{t}, i p^{t-1}}\right)$. The homology suspension maps the subspace spanned by $h_{i, j}$ 's bijectively onto $P H_{*}\left(\Omega V_{n, m}\right)$. Since the action of $P_{*}^{p^{k}}$ commutes with the homology suspension, the result follows from (4.15), (6.2) and (6.3).

Before we determine the action of $P_{*}^{p^{k}}$ on $g_{i, j}$ in $H_{*}\left(\Omega^{2} V_{n, m}\right)$, we first consider the special case $e(n, i)=0$. We denote $g_{i, j}$ by $h_{i, j}^{2}$ if $p=2$ and $e(n, i)=0$ from now on.

Theorem 6.5. If $e\left(n, i p^{t}\right)=0$, the action of $P_{*}^{p^{k}}$ on $g_{i p^{t}, j} \in H_{*}\left(\Omega^{2} V_{n, m}\right)$ ( $p \nmid i, m+1 \leqq i p^{t} \leqq n, t=0$ or $\left.i p^{t} \leqq m p, j \geqq 0\right)$ is given as follows

(i) The case $t=0$ :

$$
P_{*}^{p^{k}} g_{i, j}= \begin{cases}-g_{i, j-1}^{p}, & k=0, j \geqq 1 \\ \left(d_{i, k-j-1}+1\right) g_{i-p^{k-j-1}(p-1), j}, & k \geqq j+1, \\ & i-p^{k-j-1}(p-1)>\max \left\{m, \frac{n}{p}\right\} \\ 0, & \text { otherwise }\end{cases}
$$

(ii) The case $t>0$ :

$$
P_{*}^{p^{k}} g_{i p^{t}, j}= \begin{cases}-g_{i p^{t}, j-1}^{p}, & k=0, j \geqq 1 \\ \left(d_{i, k-j-t-1}+1\right) g_{i p^{t}-p^{k-j-1}(p-1), j}, & k>j+t, i>p^{k-j-t}, \\ & i p^{t}-p^{k-j-1}(p-1)>\max \left\{m, \frac{n}{p}\right\} \\ g_{i p^{t}-p^{k-j-1}(p-1), j}, & j+1 \leqq k \leqq j+t, \\ & i>p^{k-j-t}, \\ & i p^{t}-p^{k-j-1}(p-1)>\max \left\{m, \frac{n}{p}\right\} \\ 0, & \text { otherwise }\end{cases}
$$


Proof. By Nishida relation and (5.4), we have

$$
P_{*}^{p^{k}} g_{i p^{t}, j}= \begin{cases}-g_{i p^{t}, j-1}^{p}, & k=0, j \geqq 1 \\ 0, & k=j=0 \\ \beta \xi_{1} P_{*}^{P^{k-1} h_{i p^{t}, j},} & k \geqq 1\end{cases}
$$

if $p$ is odd. Then, (5.4) and (6.4) yield the result if $p$ is odd. If $p=2$, we can verify the result directly from (6.4).

Lemma 6.6. Let $B_{n, m}$ be the sub Hopf algebra of $H_{*}\left(\Omega^{2} V_{n, m}\right)$ generated by $\left\{g_{i, j} \mid m+1 \leqq i \leqq n, p \nmid i\right.$ or $\left.i \leqq m p, j \geqq 0\right\}$, Then, $\pi_{n, m *}: H_{*}\left(\Omega^{2} V_{n, m}\right)$ $\rightarrow H_{*}\left(\Omega^{2} V_{n, m+1}\right)$ maps $B_{n, m}$ onto $B_{n, m+1}$ and it maps $B_{n, m}$ isomorphically

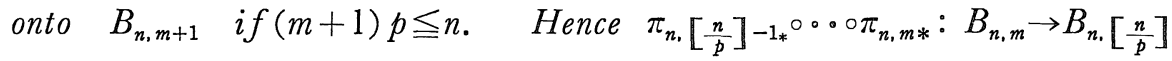
$(m p \leqq n)$ is an isomorphism and it maps $g_{i, j}$ to $g_{i p^{e(n, i)}, j}$ and $g_{k(i-1, m), j}$ to $g_{i, j \circ}$

Proof. This is immediate from (4. 11).

Since all of the even dimensional primitive elements of $H_{*}\left(\Omega^{2} V_{n, m}\right)$ are contained in $B_{n, m}, B_{n, m}$ is closed under the action of the Steenrod algebra. Hence (6.5) and (6.6) allow us to determine the action of $P_{*}^{p^{k}}$ on $g_{i, j} \in H_{*}\left(\Omega^{2} V_{n, m}\right)$ when $e(n, i)>0$.

Theorem 6.7. If $e(n, i)>0$, the action of $P_{*}^{k}$ on $g_{i, j} \in H_{*}\left(\Omega^{2} V_{n, m}\right)$ $\left(m+1 \leqq i \leqq \frac{n}{p}, p \nmid i\right.$ or $\left.i \leqq m p, j \geqq 0\right)$ is given as follows.

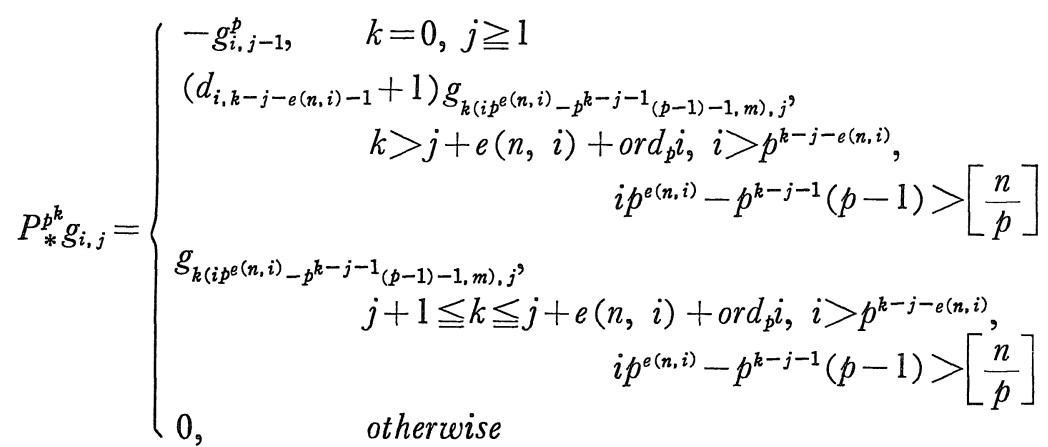

Proof. This is straightforward from (6.5) and (6.6). 


\section{References}

[1] A. Borel, Sur la cohomologie des espaces fibrés principaux et des espaces homogènes de groupes de Lie compacts, Ann. of Math., 57 (1953), 115-207.

[2] F. R. Cohen, T. J. Lada, and J. P. May, The Homology of Iterated Loop Spaces, Lecture Notes in Math. 533, Springer-Verlag, 1976.

[3] D. Husemoller, The structure of the Hopf algebra $H_{*}(B U)$ over a $Z_{(p)}$-algebra, Amer. J. Math., 93 (1971), 329-349.

[4] J. W. Milnor and J. C. Moore, On the structure of Hopf algebras, Ann. of Math., 81 (1965), 211-264.

[5] D. C. Ravenel, The structure of Morava stabilizer algebras, Inv. Math., 37 (1976), 109-120.

[6] D. C. Ravenel and W. S. Wilson, Bipolynomial Hopf algebras, J. of. Pure and Appl. Alg., 4 (1974), 41-45.

[7] A. Yamaguchi, Note on the Eilenberg-Moore spectral sequence, to appear. 